\title{
BREDON COHOMOLOGY AND ROBOT MOTION PLANNING
}

\author{
MICHAEL FARBER, MARK GRANT, GREGORY LUPTON, AND JOHN OPREA
}

\begin{abstract}
In this paper we study the topological invariant $\mathrm{TC}(X)$ reflecting the complexity of algorithms for autonomous robot motion. Here, $X$ stands for the configuration space of a system and $\mathrm{TC}(X)$ is, roughly, the minimal number of continuous rules which are needed to construct a motion planning algorithm in $X$. We focus on the case when the space $X$ is aspherical; then the number TC $(X)$ depends only on the fundamental group $\pi=\pi_{1}(X)$ and we denote it $\mathrm{TC}(\pi)$. We prove that $\mathrm{TC}(\pi)$ can be characterised as the smallest integer $k$ such that the canonical $\pi \times \pi$-equivariant map of classifying spaces

$$
E(\pi \times \pi) \rightarrow E_{\mathcal{D}}(\pi \times \pi)
$$

can be equivariantly deformed into the $k$-dimensional skeleton of $E_{\mathcal{D}}(\pi \times \pi)$. The symbol $E(\pi \times \pi)$ denotes the classifying space for free actions and $E_{\mathcal{D}}(\pi \times \pi)$ denotes the classifying space for actions with isotropy in the family $\mathcal{D}$ of subgroups of $\pi \times \pi$ which are conjugate to the diagonal subgroup. Using this result we show how one can estimate $\mathrm{TC}(\pi)$ in terms of the equivariant Bredon cohomology theory. We prove that $\mathrm{TC}(\pi) \leq \max \left\{3, \operatorname{cd}_{\mathcal{D}}(\pi \times \pi)\right\}$, where $\operatorname{cd}_{\mathcal{D}}(\pi \times \pi)$ denotes the cohomological dimension of $\pi \times \pi$ with respect to the family of subgroups $\mathcal{D}$. We also introduce a Bredon cohomology refinement of the canonical class and prove its universality. Finally we show that for a large class of principal groups (which includes all torsion free hyperbolic groups as well as all torsion free nilpotent groups) the essential cohomology classes in the sense of Farber and Mescher [17] are exactly the classes having Bredon cohomology extensions with respect to the family $\mathcal{D}$.
\end{abstract}

\section{INTRODUCTION}

The topological complexity, $\mathrm{TC}(X)$, is a numerical homotopy invariant of a path-connected topological space $X$, originally introduced in [11] (see also [15]) which is motivated by the motion planning problem of robotics. Roughly, $\mathrm{TC}(X)$ is the minimal number of continuous rules which are needed to construct an algorithm for autonomous motion planning of a system having $X$ as its configuration space.

To give more detail, assume that a system (robot) has to be programmed to move autonomously from any initial state to any final state. Let $X$ denote the configuration space of the system; points of $X$ represent states of the system and continuous paths in $X$ represent motions of the system. A motion planning algorithm is a function which

Michael Farber was partially supported by the EPSRC, by the IIAS and by the Marie Curie Actions, FP7, in the frame of the EURIAS Fellowship Programme.

Gregory Lupton and John Oprea were partially supported by grants from the Simons Foundation (\#209575 and \#244393).

This research was supported through the programme "Research in Pairs" by the Mathematisches Forschungsinstitut Oberwolfach in 2017. 
associates with any pair of states $(A, B) \in X \times X$ a continuous motion of the system starting at $A$ and ending at $B$. In other words, a motion planning algorithm is a section of the path fibration

$$
p: X^{I} \rightarrow X \times X, \quad p(\gamma)=(\gamma(0), \gamma(1))
$$

Here $X^{I}$ denotes the space of all continuous paths $\gamma: I=[0,1] \rightarrow X$ equipped with the compact-open topology. It is easy to see that the fibration (1) admits a continuous section if and only if $X$ is contractible [11]. The topological complexity $\mathrm{TC}(X)$ is an integer (see Definition 2.1.1 below) reflecting the complexity of this fibration. It has several different characterisations, see [14]. Intuitively, $\mathrm{TC}(X)$ is a measure of the navigational complexity of $X$ viewed as the configuration space of a system. $\mathrm{TC}(X)$ is similar in spirit to the classical Lusternik - Schnirelmann category cat $(X)$. The invariants $\mathrm{TC}(X)$ and cat $(X)$ are special cases of a more general notion of genus of a fibration introduced by A. Schwarz [34]. A recent survey of the concept $\mathrm{TC}(X)$ and robot motion planning algorithms in practically interesting configuration spaces can be found in [16].

One of the main properties of $\mathrm{TC}(X)$ is its homotopy invariance [11], i.e. $\mathrm{TC}(X)$ depends only on the homotopy type of $X$. This property is helpful for the task of computing $\mathrm{TC}(X)$ in various examples since cohomological tools can be employed. In the case when the configuration space $X$ is aspherical, i.e. $\pi_{i}(X)=0$ for all $i>1$, the number $\operatorname{TC}(X)$ depends only on the fundamental group $\pi=\pi_{1}(X)$ and it was observed in [14] that one should to be able to express $\mathrm{TC}(X)$ in terms of the algebraic properties of the group $\pi$ alone. This remark justifies the notation $\mathrm{TC}(\pi)$ for $\mathrm{TC}(K(\pi, 1))$.

A similar question for the Lusternik - Schnirelmann category cat $(X)$ was solved by $\mathrm{S}$. Eilenberg and T. Ganea in 1957 in the seminal paper [10]. Their theorem relates cat $(X)$ and the cohomological dimension of the fundamental group $\pi$ of $X$.

The problem of computing $\mathrm{TC}(\pi)$ as an algebraic invariant of the group $\pi$ has attracted the attention of many mathematicians and many interesting partial results have been obtained. It is easy to see that $\mathrm{TC}(\pi)=\infty$ if $\pi$ has torsion; therefore we shall always restrict our attention to torsion free groups $\pi$.

The initial papers [11], [14] contained computations of $\mathrm{TC}(X)$ for graphs, closed orientable surfaces and tori. In [18] the number $\operatorname{TC}(X)$ was computed for the case when $X$ is the configuration space of many particles moving on the plane without collisions. D. Cohen and G. Pruidze [4] calculated the topological complexity of complements of general position arrangements and Eilenberg - MacLane spaces associated to certain right-angled Artin groups.

In a recent breakthrough, the topological complexity of closed non-orientable surfaces of genus $g \geq 2$ was computed by A. Dranishnikov for $g \geq 4$ in [9] and by D. Cohen and L. Vandembroucq for $g=2,3$ in [5]. In both these articles it is shown that $\mathrm{TC}(\pi)$ attains its maximum, i.e. coincides with $\operatorname{cd}(\pi \times \pi)$, the cohomological dimension of the group $\pi \times \pi$.

The estimates of M. Grant [20] give good upper bounds for $\mathrm{TC}(\pi)$ for nilpotent fundamental groups $\pi$. In [21], M. Grant, G. Lupton and J. Oprea proved that TC $(\pi)$ is bounded below by the cohomological dimension of $A \times B$ where $A$ and $B$ are subgroups of $\pi$ whose 
conjugates intersect trivially. Using these estimates, M. Grant and D. Recio-Mitter [24] have computed TC $(\pi)$ for certain subgroups of Artin's braid groups.

Y. Rudyak [33] went in the opposite direction by showing that for any pair of positive integers $k, \ell$ satisfying $k \leq \ell \leq 2 k$ there exists a finitely presented group $\pi$ such that $\operatorname{cd}(\pi)=k$ and $\mathrm{TC}(\pi)=\ell$.

In a recent preprint [17] M. Farber and S. Mescher showed that for a large class of groups (including all torsion free hyperbolic groups) the topological complexity TC $(\pi)$ equals either $\operatorname{cd}(\pi \times \pi)$ or $\operatorname{cd}(\pi \times \pi)-1$. Since hyperbolic groups are typical in many models of random groups this gives an answer with possible error 1 for a typical group. Note that $\operatorname{cd}(\pi \times \pi)$ is obviously an upper bound for $\mathrm{TC}(\pi)$ for any $\pi$.

In this paper we tackle the general problem of understanding $\mathrm{TC}(\pi)$ from a different direction, using the tools of equivariant topology. We are not interested in computing examples, but rather in reformulating the problem itself so that interactions with subjects such as group theory and homological algebra become apparent. This re-interpretation, together with previously computed examples of $\mathrm{TC}(\pi)$, provides illustrative examples for Bredon cohomology with respect to a family of subgroups.

Firstly we reduce the problem to a question about classifying spaces of families of subgroups. Namely, we define a special class $\mathcal{D}$ of subgroups of $G=\pi \times \pi$ and prove that the number $\mathrm{TC}(\pi)$ coincides with the smallest $k$ such that the canonical map of classifying spaces

$$
E(G) \rightarrow E_{\mathcal{D}}(G)
$$

can be factored through a $G$-CW-complex of dimension $\leq k$. Here $E(G)$ is the classical classifying space for free $G$-actions and $E_{\mathcal{D}}(G)$ is the classifying space for $G$-actions with isotropy subgroups in the class $\mathcal{D}$. Specifically, $\mathcal{D}$ consists of all subgroups $H \subset \pi \times \pi$ which are conjugate to the diagonal subgroup. Using this reduction we establish an upper bound

$$
\mathrm{TC}(\pi) \leq \max \left\{3, \operatorname{cd}_{\mathcal{D}}(\pi \times \pi)\right\}
$$

where $\operatorname{cd}_{\mathcal{D}}(\pi \times \pi)$ denotes the cohomological dimension of $\pi \times \pi$ with respect to the family $\mathcal{D}$. Secondly, we use Bredon cohomology to produce lower bounds for $\mathrm{TC}(\pi)$. Namely we show that if (for some $\mathcal{O}_{\mathcal{D}}$-module $\underline{M}$ ) there exists a Bredon cohomology class

$$
\underline{\alpha} \in H_{\mathcal{D}}^{n}(\pi \times \pi ; \underline{M})
$$

such that the cohomology class

$$
\Phi(\underline{\alpha}) \neq 0 \in H^{n}(\pi \times \pi ; M)
$$

is nonzero, then $\mathrm{TC}(X) \geq n$. Here $M$ denotes the principal component of $\underline{M}$ and

$$
\Phi: H_{\mathcal{D}}^{n}(\pi \times \pi ; \underline{M}) \rightarrow H^{n}(\pi \times \pi ; M)
$$

is a natural homomorphism from the Bredon cohomology to the usual twisted cohomology. The notions we use here are explained in full detail in the sequel.

We define a Bredon cohomology generalisation of the canonical class $\mathfrak{u} \in H_{\mathcal{D}}^{1}(\pi \times \pi ; \underline{I})$ which refines the canonical class $\mathfrak{v} \in H^{1}(\pi \times \pi ; I)$ introduced in [6]. We prove a universality 
theorem for the powers of the Bredon canonical class $\mathfrak{u}$ which implies, in particular, that

$$
\operatorname{cd}_{\mathcal{D}}(\pi \times \pi)=\operatorname{height}(\mathfrak{u})
$$

(the height of a cohomology class is the exponent of the maximal non-vanishing power).

In [17], Farber and Mescher introduced the notion of an essential cohomology class as a class $\beta \in H^{n}(\pi \times \pi ; A)$ which can be obtained via a coefficient homomorphism $\mu: I^{n} \rightarrow A$ from the power $\mathfrak{v}^{n}$ of the canonical class, i.e. $\beta=\mu_{*}\left(\mathfrak{v}^{n}\right)$. In this paper we introduce a class of principal groups $\pi$ and we show that for principal groups a cohomology class in $H^{n}(\pi \times \pi ; M)$ is in the image of the map $\Phi$ from Bredon cohomology if and only if it is essential. We also prove that the class of principal groups includes all torsion free hyperbolic groups and all torsion free nilpotent groups.

Curiously, the fundamental group of the Klein bottle is not principal (see Example 8.0.7) but nevertheless for this group

$$
\mathrm{TC}(\pi)=\operatorname{height}(\mathfrak{v})
$$

as follows from the theorem of D. Cohen and L. Vandembroucq [5].

While results of this paper are more conclusive for $\mathrm{TC}(\pi) \geq 3$, we mention that $\mathbb{Z}$ is the only group satisfying $\mathrm{TC}(\pi)=1$ (as follows from [22]) and groups with $\mathrm{TC}(\pi)=2$ are likely quite restricted, see [1]. The obvious examples of groups $\pi$ with $\operatorname{TC}(\pi)=2$ include $\mathbb{Z}^{2}$ and the non-commutative free group $F$.

\section{THE FIRST REDUCTION}

2.1. The concept of topological complexity. We start by recalling the definition of the invariant $\mathrm{TC}(X)$.

Definition 2.1.1. Given a path-connected topological space $X$, the topological complexity of $X$ is the minimal integer $\mathrm{TC}(X)=k$ such that the Cartesian product $X \times X$ can be covered by $k+1$ open subsets

$$
X \times X=U_{0} \cup U_{1} \cup \cdots \cup U_{k}
$$

with the property that for any $i=0,1,2, \ldots, k$ there exists a continuous section of the fibration (1)

$$
s_{i}: U_{i} \rightarrow X^{I}, \quad p \circ s_{i}=\operatorname{incl}_{U_{i}}
$$

over $U_{i}$. If no such $k$ exists we will set $\mathrm{TC}(X)=\infty$.

Note that in this paper we are using the reduced version of $\operatorname{TC}(X)$ which is one less than the original notion used in [11], [15], [16] and [17].

For convenience of the reader we also recall the notion of the Schwarz genus of a fibration (also known as the sectional category).

Definition 2.1.2. Let $p: E \rightarrow B$ be a Serre fibration over a path-connected topological space $B$. The Schwarz genus of $p$ is defined as the smallest integer $k$ such that the base $B$ admits an open cover $B=U_{0} \cup U_{1} \cup \cdots \cup U_{k}$ such that the fibration $p$ admits a continuous section over $U_{i}$ for each $i=0,1, \ldots, k$. 
This paper is mainly dedicated to the problem of computing $\operatorname{TC}(X)$ in the case when $X$ is an aspherical finite cell complex. Recall that a connected cell complex $X$ is said to be aspherical if $\pi_{i}(X)=0$ for all $i>1$. The notation $X=K(\pi, 1)$ means that $X$ is aspherical and its fundamental group is $\pi$. A key property of $\mathrm{TC}(X)$ is its homotopy invariance, see [11]. The homotopy invariance of the topological complexity implies that the number

$$
\mathrm{TC}(\pi)=\mathrm{TC}(K(\pi, 1))
$$

depends only on the group $\pi$.

2.2. Many systems of practical interest have aspherical configuration spaces. Consider for example the problem of coordinated collision free motion planning of a set of objects on the plane $\mathbf{R}^{2}$. We may represent the objects by discs of radius $r>0$ and the state of each disc is determined by the position of its centre $A_{i} \in \mathbf{R}^{2}$ where $i=1, \ldots, n$. Thus a state of the system is a configuration of points $\left(A_{1}, A_{2}, \ldots, A_{n}\right)$, where $A_{i} \in \mathbf{R}^{2}$, such that

$$
\left|A_{i}-A_{j}\right|>2 r, \quad i \neq j \text {. }
$$

Let $F_{r}\left(\mathbf{R}^{2}, n\right)$ denote the configuration space of this system. It is common to relax the problem and consider instead the weaker condition

$$
A_{i} \neq A_{j}, \quad i \neq j
$$

which leads to the usual configuration space $F\left(\mathbf{R}^{2}, n\right)$ of $n$ distinct points on the plane. It is easy to see that $F_{r}\left(\mathbf{R}^{2}, n\right)$ and $F\left(\mathbf{R}^{2}, n\right)$ are homeomorphic and, moreover, it is well known that the space $F\left(\mathbf{R}^{2}, n\right)$ is aspherical as can be seen using the tower of Fadell-Neuwirth fibrations.

2.3. Consider a continuous partial section $s: U \rightarrow X^{I}$ of the fibration (1) over a subset $U \subset X \times X$. Using the exponential correspondence, the map $s$ can be viewed as a homotopy $h: U \times I \rightarrow X$ where $h(u, t)=s(u)(t)$ for $u \in U, t \in I$. Let $p_{j}: X \times X \rightarrow X$ (where $j=1,2$ ) denote the projections onto the first and the second factors. The property of $s$ being a section can be expressed by saying that the homotopy $h$ connects the projections of $U$ onto the first and second coordinates, i.e. $h(u, 0)=p_{1}(u)$ and $h(u, 1)=p_{2}(u)$.

Thus we see that the open sets $U_{i} \subset X \times X$ which appear in Definition 2.1.1 can be equivalently characterised by the property that their two projections $U_{i} \rightarrow X$ on the first and the second factors are homotopic.

In the case when the space $X$ is aspherical we can use the following property: For a connected space $U$ that is homotopy equivalent to a cell complex, the set of homotopy classes of maps $U \rightarrow X$ is in a one-to-one correspondence with the set of conjugacy classes of homomorphisms $\pi_{1}\left(U, u_{0}\right) \rightarrow \pi_{1}\left(X, x_{0}\right)$, see Chapter V, Corollary 4.4 in [36]. Recall that two group homomorphisms $f, g: \pi_{1}\left(U, u_{0}\right) \rightarrow \pi_{1}\left(X, x_{0}\right)$ are conjugate if there exists $\beta \in \pi_{1}\left(X, x_{0}\right)$ such that for all $\alpha \in \pi_{1}\left(U, u_{0}\right)$ one has $f(\alpha)=\beta g(\alpha) \beta^{-1}$.

These remarks lead to the following definition:

Definition 2.3.1. Let $X$ be a path-connected topological space with fundamental group $\pi=\pi_{1}\left(X, x_{0}\right)$. The $\mathcal{D}$-topological complexity, $\operatorname{TC}^{\mathcal{D}}(X)$, is defined as the minimal number $k$ such that $X \times X$ can be covered by $k+1$ open subsets $X \times X=U_{0} \cup U_{1} \cup \cdots \cup U_{k}$ with 
the property that for any $i=0,1,2, \ldots, k$ and for every choice of the base point $u_{i} \in U_{i}$ the homomorphism $\pi_{1}\left(U_{i}, u_{i}\right) \rightarrow \pi_{1}\left(X \times X, u_{i}\right)$ induced by the inclusion $U_{i} \rightarrow X \times X$ takes values in a subgroup conjugate to the diagonal $\Delta \subset \pi \times \pi$.

Recall that there is an isomorphism $\pi_{1}\left(X \times X, u_{i}\right) \rightarrow \pi_{1}\left(X \times X,\left(x_{0}, x_{0}\right)\right)=\pi \times \pi$ determined uniquely up to conjugation, and the diagonal inclusion $X \rightarrow X \times X$ induces the inclusion $\pi \rightarrow \pi \times \pi$ onto the diagonal $\Delta$.

Lemma 2.3.2. One has $\operatorname{TC}^{\mathcal{D}}(X)=\mathrm{TC}(X)$ if $X$ is a finite aspherical cell complex.

Proof. It follows from the remarks given in $\S 2.3$. Here we use the known fact that an open subset of a finite CW-complex is homotopy equivalent to a countable CW-complex. Indeed, by Theorem 1 of J. Milnor [32], a space is homotopy equivalent to a countable $\mathrm{CW}$-complex if and only if it is homotopy equivalent to an absolute neighbourhood retract (ANR). Any finite CW-complex is an ANR and an open subset of an ANR is an ANR. Thus, an open subset of a finite CW-complex is an ANR and hence has the homotopy type of a countable CW-complex.

Lemma 2.3.3. Let $X$ be a finite aspherical cell complex with fundamental group $\pi=$ $\pi_{1}\left(X, x_{0}\right)$. Let $q: \widehat{X \times X} \rightarrow X \times X$ be the connected covering space corresponding to the diagonal subgroup

$$
\Delta \subset \pi \times \pi=\pi_{1}\left(X \times X,\left(x_{0}, x_{0}\right)\right) .
$$

Then the $\mathcal{D}$-topological complexity $\operatorname{TC}^{\mathcal{D}}(X)$ coincides with the Schwarz genus of $q$.

Proof. For an open subset $U \subset X \times X$, the condition that the induced map $\pi_{1}(U, u) \rightarrow$ $\pi_{1}(X \times X, u)$ takes values in a subgroup conjugate to the diagonal $\Delta$ is equivalent to the condition that $q$ admits a continuous section over $U$. Using this remark the Lemma follows by comparing the definitions of $\mathrm{TC}^{\mathcal{D}}(X)$ and of Schwarz genus.

Remark 2.3.4. If we remove the assumption that $X$ is aspherical then the topological complexity $\mathrm{TC}(X)$ is greater than or equal to the Schwarz genus of $q$, see [13], Theorem 4.1 .

Next we introduce terminology and notations which will be used in the statement of Theorem 2.1.

2.4. Recall that the join $X * Y$ of topological spaces $X$ and $Y$ can be defined as the quotient of the product $X \times[0,1] \times Y$ with respect to the equivalence relation $(x, 0, y) \sim\left(x, 0, y^{\prime}\right)$ and $(x, 1, y) \sim\left(x^{\prime}, 1, y\right)$ for all $x, x^{\prime} \in X$ and $y, y^{\prime} \in Y$. We have an obvious embedding $X \rightarrow X * Y$ given by $x \mapsto(x, 0, y)$ where $y \in Y$ is arbitrary.

One may use the following notation. A point $(x, t, y) \in X \times[0,1] \times Y / \sim$ can be written as a formal linear combination $(1-t) x+t y$. This notation is clearly consistent with the identifications of the join.

Let $\Delta^{k}$ denote the standard $k$-dimensional simplex, i.e.

$$
\Delta^{k}=\left\{\left(t_{0}, t_{1}, \ldots, t_{k}\right) ; t_{i} \geq 0, \quad \sum_{i=0}^{k} t_{i}=1\right\} .
$$


We may define the multiple join $X_{0} * X_{1} * \cdots * X_{k}$ of topological spaces $X_{0}, \ldots, X_{k}$ as the quotient of the product $\left(\prod_{i=0}^{k} X_{i}\right) \times \Delta^{k}$ with respect to an equivalence relation $\sim$ described below. The points of the join are written as formal linear combinations

$$
x=t_{0} x_{0}+t_{1} x_{1}+\cdots+t_{k} x_{k}, \quad x_{i} \in X_{i}, \quad\left(t_{0}, t_{1}, \ldots, t_{k}\right) \in \Delta^{k},
$$

and we say that $x \sim x^{\prime}$ where $x^{\prime}=t_{0}^{\prime} x_{0}^{\prime}+t_{1}^{\prime} x_{1}^{\prime}+\cdots+t_{k}^{\prime} x_{k}^{\prime}$ iff $t_{i}=t_{i}^{\prime}$ for all $i=0, \ldots, k$ and $x_{i}=x_{i}^{\prime}$ provided $t_{i} \neq 0$.

2.5. Let $\pi$ be a discrete group. We shall view $\pi$ as a discrete topological space with the following left $\pi \times \pi$-action:

$$
(x, y) \cdot g=x g y^{-1} .
$$

This action is transitive and the isotropy subgroup of the unit element $1 \in \pi$ coincides with the diagonal subgroup $\Delta \subset \pi \times \pi$. The isotropy subgroups of the other elements are the conjugates of $\Delta$.

2.6. For an integer $k \geq 0$, let $E_{k}(\pi)$ denote the $(k+1)$-fold join

$$
E_{k}(\pi)=\pi * \pi * \cdots * \pi .
$$

We shall equip $E_{k}(\pi)$ with the left diagonal $\pi \times \pi$-action determined by the $\pi \times \pi$-action on $\pi$ as in $\S 2.5$ above. Each $E_{k}(\pi)$ is naturally a $k$-dimensional equivariant simplicial complex with $k$-dimensional simplexes in 1-1 correspondence with sequences $\left(g_{0}, g_{1}, \ldots, g_{k}\right)$ of group elements $g_{i} \in \pi$. Note that $E_{k}(\pi)$ is $(k-1)$-connected and is in fact homotopy equivalent to a wedge of $k$-dimensional spheres.

2.7. There is a natural equivariant embedding

$$
E_{k}(\pi) \hookrightarrow E_{k+1}(\pi)=E_{k}(\pi) * \pi .
$$

Using it we may define the simplicial complex

$$
E(\pi)=\bigcup_{k=0}^{\infty} E_{k}(\pi)=\pi * \pi * \pi * \ldots,
$$

the join of infinitely many copies of $\pi$.

2.8. Furthermore, let $E(\pi \times \pi)$ denote the classical classifying space for free $\pi \times \pi$ actions, i.e.

$$
E(\pi \times \pi)=(\pi \times \pi) *(\pi \times \pi) * \ldots,
$$

the join of infinitely many copies of $\pi \times \pi$. We shall view each copy of $\pi \times \pi$ as a discrete topological space with the left free action of $\pi \times \pi$ given by $(x, y) \cdot(g, h)=(x g, y h)$ for $x, y, g, h \in \pi$. The space $E(\pi \times \pi)$ inherits the diagonal action of the group $\pi \times \pi$. 
2.9. The map $F: \pi \times \pi \rightarrow \pi$ given by $F(x, y)=x y^{-1}$ is $\pi \times \pi$-equivariant. The natural extension of $F$ to the infinite joins defines a $\pi \times \pi$-equivariant map

$$
F: E(\pi \times \pi) \rightarrow E(\pi) .
$$

Theorem 2.1. Let $X$ be a finite aspherical cell complex and let $\pi=\pi_{1}\left(X, x_{0}\right)$ be its fundamental group. Then $\mathrm{TC}(X)$ coincides with the smallest integer $k$ such that there exists a $\pi \times \pi$-equivariant map $E(\pi \times \pi) \rightarrow E_{k}(\pi)$.

Proof. Let $p: \tilde{X} \rightarrow X$ denote the universal cover of $X$. Here $\tilde{X}$ is an equivariant cell complex with free left $\pi$-action. The map $p \times p: \tilde{X} \times \tilde{X} \rightarrow X \times X$ is the universal cover of $X \times X$. We shall view $p \times p$ as a principal $G=\pi \times \pi$-bundle and for $k=0,1, \ldots$ construct the associated bundle

$$
q_{k}:(\tilde{X} \times \tilde{X}) \times{ }_{G} E_{k}(\pi) \rightarrow X \times X .
$$

Here $(\tilde{X} \times \tilde{X}) \times{ }_{G} E_{k}(\pi)$ denotes the quotient of the product $(\tilde{X} \times \tilde{X}) \times E_{k}(\pi)$ with respect to the following $G=\pi \times \pi$-action: $(g, h) \cdot\left(x, x^{\prime}, z\right)=\left(g x, h x^{\prime},(g, h) \cdot z\right)$ where $g, h \in \pi$, $x, x^{\prime} \in \tilde{X}$ and $z \in E_{k}(\pi)$.

First we observe that the fibration $q_{0}$ coincides with the covering space $q: \widehat{X \times X} \rightarrow$ $X \times X$ corresponding to the diagonal subgroup $\Delta \subset \pi \times \pi$ which appears in Lemma 2.3.3. Indeed, $E_{0}(\pi)=\pi$ has a transitive $G=\pi \times \pi$-action and the isotropy of the unit element $1 \in \pi$ is the diagonal $\Delta \subset G=\pi \times \pi$. Hence we obtain a homeomorphism

$$
\left(\tilde{X} \times \tilde{X} \times E_{0}(\pi)\right) / G \rightarrow(\tilde{X} \times \tilde{X}) / \Delta
$$

commuting with the projections onto $X \times X$; thus we see that the fibration $q_{0}$ is isomorphic to the fibration $p \times p:(\tilde{X} \times \tilde{X}) / \Delta \rightarrow X \times X$. It is obvious that the latter fibration is isomorphic to the connected covering $q$ corresponding to the diagonal subgroup $\Delta \subset \pi \times \pi$.

Applying Lemma 2.3.2 and Lemma 2.3.3 we obtain that $\mathrm{TC}(X)$ coincides with the Schwarz genus of the fibration $q_{0}$.

Next we apply a theorem of A. Schwarz (see [34], Theorem 3) stating that genus of a fibration $p: E \rightarrow B$ equals the smallest integer $k$ such that the fiberwise join $p * p * \cdots * p$ of $k+1$ copies of the fibration $p: E \rightarrow B$ admits a continuous section. The fiberwise join of $k+1$ copies of the fibration $q_{0}$ coincides with the fibration $q_{k}$. Thus we obtain that $\operatorname{TC}(X)$ coincides with the smallest $k$ such that $q_{k}$ has a continuous section.

Finally we apply Theorem 8.1 from [26], chapter 4, which states that continuous sections of the fibre bundle $q_{k}$ are in 1-1 correspondence with $G=\pi \times \pi$-equivariant maps

$$
\tilde{X} \times \tilde{X} \rightarrow E_{k}(\pi) .
$$

Thus, $\mathrm{TC}(X)$ is the smallest $k$ such that a $G=\pi \times \pi$-equivariant map (6) exists. Finally we observe that the space $\tilde{X} \times \tilde{X}$ is $G=\pi \times \pi$-equivariantly homotopy equivalent to $E(\pi \times \pi)$ (in view of the Milnor construction) and the result follows.

\section{THE SECOND REDUCTION}

In this section we prove the following statement which gives an intrinsic version of Theorem 2.1. 
Theorem 3.1. Let $X$ be a finite aspherical cell complex and let $\pi=\pi_{1}\left(X, x_{0}\right)$ be its fundamental group. Then $\mathrm{TC}(X)$ coincides with the minimal dimension of a $\pi \times \pi-C W$ complex $L$ such that the map $F$ (see (4)), viewed up to $\pi \times \pi$-equivariant homotopy, can be $\pi \times \pi$-equivariantly factored as follows:

$$
E(\pi \times \pi) \rightarrow L \rightarrow E(\pi)
$$

The proof of Theorem 3.1 will follow a brief review of the basic material concerning classifying spaces and families of subgroups; we shall mainly follow [27].

3.1. Let $G$ be a discrete group. $A G$-CW-complex is a CW-complex $X$ with a left $G$-action such that for each open cell $e \subset X$ and each $g \in G$ with $g e \cap e \neq \emptyset$, the left multiplication by $g$ acts identically on $e$.

A simplicial complex with a simplicial $G$-action is a $G$-CW-complex (with respect to the barycentric subdivision), see [27], Example 1.5.

A family $\mathcal{F}$ of subgroups of $G$ is a set of subgroups of $G$ which is closed under conjugation and finite intersections.

3.2. A classifying $G$-CW-complex $E_{\mathcal{F}}(G)$ with respect to a family $\mathcal{F}$ of subgroups of $G$ is defined as a $G$-CW-complex $E_{\mathcal{F}}(G)$ such that

(a) the isotropy subgroup of any element of $E_{\mathcal{F}}(G)$ belongs to $\mathcal{F}$;

(b) For any $G$-CW-complex $Y$ all of whose isotropy subgroups belong to $\mathcal{F}$ there is up to $G$-homotopy exactly one $G$-map $Y \rightarrow E_{\mathcal{F}}(G)$.

A $G$-CW-complex $X$ is a model for $E_{\mathcal{F}}(G)$ if and only if all its isotropy subgroups belong to the family $\mathcal{F}$ and for each $H \in \mathcal{F}$ the set of $H$-fixed points $X^{H}$ is weakly contractible, i.e. $\pi_{i}\left(X^{H}, x_{0}\right)=0$ for any $i=0,1, \ldots$ and for any $x_{0} \in X^{H}$. See [27], Theorem 1.9.

3.3. We shall use below the equivariant version of the Whitehead Theorem which we shall state as follows (see [30], Theorem 3.2 in Chapter 1).

Theorem 3.2 (Whitehead theorem). Let $f: Y \rightarrow Z$ be a $G$-map between $G$ - $C W$-complexes such that for each subgroup $H \subset G$ the induced map $\pi_{i}\left(Y^{H}, x_{0}\right) \rightarrow \pi_{i}\left(Z^{H}, f\left(x_{0}\right)\right)$ is an isomorphism for $i<k$ and an epimorphism for $i=k$ for any base point $x_{0} \in Y^{H}$. Then for any $G$-CW-complex $X$ the induced map on the set of $G$-homotopy classes

$$
f_{*}:[X, Y]_{G} \rightarrow[X, Z]_{G}
$$

is an isomorphism if $\operatorname{dim} X<k$ and an epimorphism if $\operatorname{dim} X \leq k$.

Example 3.3.1. The following example of a family of subgroups will play an important role in this paper. Let $\pi$ be a discrete group. Consider $G=\pi \times \pi$ and let $\Delta \subset \pi \times \pi$ denote the diagonal, $\Delta=\{(g, g) ; g \in \pi\}$. We shall denote by $\mathcal{D}$ the family of subgroups of $G$ generated by $\Delta$ and by the trivial subgroup $\{1\}$; in other words, $\mathcal{D}$ is the minimal family of subgroups of $G$ containing $\{1\}$ and $\Delta$. Besides the diagonal $\Delta$, the family $\mathcal{D}$ contains all the conjugates of $\Delta$ and all their finite intersections. A conjugate of $\Delta$ has the form

$$
\Delta_{c}=\left\{\left(g, c^{-1} g c\right) ; g \in \pi\right\}
$$


i.e. it is the graph of a conjugation homomorphism $g \mapsto c^{-1} g c$, where $g \in \pi$. If $\left\{c_{1}, \ldots, c_{k}\right\}$ is a finite set of elements of $\pi$ then the intersection of the corresponding subgroups $\cap_{i=1}^{k} \Delta_{c_{i}}$ can be described as follows. Let $S \subset \pi$ denote the set of all elements $c_{i} c_{j}^{-1}$, where $i, j=$ $1, \ldots, k$, and let $Z(S) \subset \pi$ denote the centraliser of $S$, i.e. the set of all elements $g \in \pi$ which commute with each element of $S$. Then

$$
\bigcap_{i=1}^{k} \Delta_{c_{i}}=\left\{\left(g, c g c^{-1}\right) ; g \in Z(S)\right\}, \quad c=c_{1} .
$$

3.4. Proof of Theorem 3.1. First note that $G=\pi \times \pi$ acts freely on $E(\pi \times \pi)$ which is the classifying $G$-CW-complex for free $G$-actions (the Milnor construction). We refer to Example 1.5 from [27] which implies that $E(\pi \times \pi)$ is a $G$-CW-complex.

Next we examine the isotropy subgroups of $G=\pi \times \pi$ acting on $E_{k}(\pi)$ and $E(\pi)$. Recall that $G$ acts on $\pi$ according to formula (3). The isotropy of an element $c \in \pi$ is the subgroup $\Delta_{c}=\left\{\left(g, c^{-1} g c\right) ; g \in \pi\right\} \subset \pi \times \pi$ which is conjugate to the diagonal subgroup $\Delta$.

It is easy to see that for a subgroup $H \subset G$ the fixed point set $\pi^{H}$ is non-empty iff $H$ is contained in a subgroup conjugate to the diagonal $\Delta \subset G$.

For an element $x \in E_{k}(\pi)$,

$$
x=t_{0} x_{0}+t_{1} x_{1}+\cdots+t_{k} x_{k},
$$

where $x_{i} \in \pi, t_{i} \in(0,1], i=0,1, \ldots, k, t_{0}+t_{1}+\cdots+t_{k}=1$, the isotropy subgroup is the intersection of the isotropy subgroups of the elements $x_{i}$. This intersection can be presented as follows. Let $S$ denote the set $\left\{x_{i} x_{j}^{-1} ; i, j=0,1, \ldots, k\right\}$. As in Example 3.3.1 the symbol $Z(S)$ denotes the centraliser of $S$, i.e. the set of all $a \in \pi$ which commute with any element of $S$. Then the isotropy subgroup of $x$ equals

$$
H_{b, S}=\left\{\left(a, b a b^{-1}\right) ; a \in Z(S)\right\}
$$

where $b=x_{i}^{-1}$ for any $i=0,1, \ldots, k$.

If $H \subset \pi \times \pi$ is a subgroup contained in a subgroup of type (8), i.e. $H \subset H_{b, S}$, then the set $\pi^{H}$ is not empty and

$$
E_{k}(\pi)^{H}=\pi^{H} * \pi^{H} * \cdots * \pi^{H}, \quad(k+1 \quad \text { times }) .
$$

We see that the space $E_{k}(\pi)^{H}$ is nonempty and is $(k-1)$-connected. At the same time the space $E(\pi)^{H}=\pi^{H} * \pi^{H} * \cdots$ (the infinite join) is non-empty and contractible. We will use this property below in order to invoke the Whitehead theorem.

As in Example 3.3.1 we denote by $\mathcal{D}$ the family of subgroups of $\pi \times \pi$ containing the trivial subgroup and the groups $H_{b, S}$, for all $b \in \pi$ and all finite subsets $S \subset \pi$.

The above discussion shows that $E(\pi)$ is the classifying $G$-CW-complex $E_{\mathcal{D}}(G)$ with respect to the family $\mathcal{D}$, see $\S 3.2$. In particular, we obtain that any two $G$-maps $X \rightarrow E(\pi)$ are $G$-homotopic provided all isotropy subgroups of $X$ are in $\mathcal{D}$.

Let $k_{1}$ denote the minimal $k$ such that there exists an equivariant map $E(\pi \times \pi) \rightarrow E_{k}(\pi)$. We know that $k_{1}=\mathrm{TC}(X)$ by Theorem 2.1. Let $k_{2}$ be the smallest dimension of a $G$-CW complex $L$ admitting a factorisation (7). We have $k_{2} \leq k_{1} \operatorname{since} \operatorname{dim} E_{k}(\pi)=k$ and any 
two equivariant maps $E(G) \rightarrow E(\pi)$ are equivariantly homotopic. On the other hand, suppose we have

$$
E(\pi \times \pi) \stackrel{\alpha}{\rightarrow} L \stackrel{\beta}{\rightarrow} E(\pi)
$$

with $\operatorname{dim} L \leq k$ and $\alpha, \beta$ being $G$-maps. We may apply the Whitehead Theorem 3.2 to the inclusion $E_{k}(\pi) \rightarrow E(\pi)$ concluding that for any $G$-CW-complex $L$ of dimension $\leq k$ the map

$$
\left[L, E_{k}(\pi)\right]_{G} \rightarrow[L, E(\pi)]_{G}
$$

is surjective. We then obtain a $G$-map $g: L \rightarrow E_{k}(\pi)$ and its composition $g \circ \alpha: E(\pi \times \pi) \rightarrow$ $E_{k}(\pi)$; clearly the composition $E(\pi \times \pi) \stackrel{g \circ \alpha}{\rightarrow} E_{k}(\pi) \hookrightarrow E(\pi)$ is $G$-homotopic to $\beta \circ \alpha$. This shows that $k_{1} \leq k_{2}$ and hence $k_{1}=k_{2}$ proving Theorem 3.1.

We can restate Theorem 3.1 as follows:

Theorem 3.3. Let $X$ be a finite aspherical cell complex and let $\pi=\pi_{1}\left(X, x_{0}\right)$ be its fundamental group. Let $G$ denote the group $\pi \times \pi$. Then $\mathrm{TC}(X)$ coincides with the minimal integer $k$ such that the canonical map

$$
E(G) \rightarrow E_{\mathcal{D}}(G)
$$

is $G$-equivariantly homotopic to a map with values in the $k$-dimensional skeleton $E_{\mathcal{D}}(G)^{(k)}$.

In the statement of Theorem 3.3 by a canonical map we mean a G-equivariant map whose existence and uniqueness (up to G-homotopy) is stated in subsection 3.2, (b).

Proof. If the map (9) is $G$-homotopic to a map with values in $E_{\mathcal{D}}(G)^{(k)}$ then we can take $L=E_{\mathcal{D}}(G)^{(k)}$ to obtain a factorisation of Theorem 3.1. Conversely, given a factorisation of Theorem 3.1, the map $L \rightarrow E_{\mathcal{D}}(G)$ can be deformed into $E_{\mathcal{D}}(G)^{(k)}$ using the $G$-cellular approximation theorem.

Let us recall that the Lusternik - Schnirelmann category of an aspherical space can be characterised in a similar way:

Proposition 3.4.1. Let $X$ be a finite aspherical cell complex and let $\pi=\pi_{1}\left(X, x_{0}\right)$ be its fundamental group. Then the Lusternik - Schnirelmann category cat $(X)$ coincides with the minimal dimension of a $\pi-C W$ complex $L$ such that the identity map $E(\pi) \rightarrow E(\pi)$ can be $\pi$-equivariantly factored as follows

$$
E(\pi) \rightarrow L \rightarrow E(\pi)
$$

This statement is essentially contained in [10], compare [10, Proposition 1] where, however there is an assumption $n \geq 2$. The proof of Proposition 3.4.1 in the general case can be obtained similarly to the proof of Theorem 3.3 and we shall briefly indicate the main steps. Firstly, one states that cat $(X)$ equals the Schwarz genus of the universal covering $\tilde{X} \rightarrow X$, compare Lemma 2.3.2 and Lemma 2.3.3. Secondly, using the theorem of Schwarz about joins we obtain that cat $(X)$ equals the smallest $k$ such that the fibration $\tilde{X} \times{ }_{\pi} E_{k}(\pi) \rightarrow X$ admits a continuous section, compare the proof of Theorem 2.1. Here we view the complex $E_{k}(\pi)$ with the left $\pi$-action which is free. Thirdly, we find that cat $(X)$ equals the smallest $k$ such that there exists a $\pi$-equivariant map $\tilde{X} \rightarrow E_{k}(\pi)$, compare Theorem 2.1 . 
And finally, one uses the universal properties of the classifying space $E(\pi)=\tilde{X}$ and the equivariant Whitehead theorem to restate the result in the form of Proposition 3.4.1.

3.5. Let $\mathcal{O}_{\mathcal{D}}$ denote the orbit category with respect to the family $\mathcal{D}$, see [2]; we shall recall these notions in the following section. Let $\operatorname{cd}_{\mathcal{D}}(\pi \times \pi)$ denote the cohomological dimension of the constant $\mathcal{O}_{\mathcal{D}}$-module $\underline{\mathbb{Z}}$. Since $E(\pi)$ is a model for the classifying space $E_{\mathcal{D}}(G)$, applying Theorem 5.2 from [27] we obtain that $E(\pi)$ has the equivariant homotopy type of a $G$-CW-complex of dimension $\leq \max \left\{3, \operatorname{cd}_{\mathcal{D}}(\pi \times \pi)\right\}$. Together with Theorem 3.1 this gives the following Corollary.

Corollary 3.5.1. Let $X$ be a finite aspherical cell complex and let $\pi=\pi_{1}\left(X, x_{0}\right)$ be its fundamental group. Then

$$
\mathrm{TC}(X) \leq \max \left\{3, \operatorname{cd}_{\mathcal{D}}(\pi \times \pi)\right\} .
$$

Proposition 3.5.2. For any discrete group $\pi$ we have $\operatorname{cd}(\pi) \leq \operatorname{cd}_{\mathcal{D}}(\pi \times \pi)$.

Proof. Recall that $G$ denotes $\pi \times \pi$. Assume first that $k:=\operatorname{cd}_{\mathcal{D}}(G) \geq 3$, so that there exists a $k$-dimensional model for $E_{\mathcal{D}}(G)$. Since the trivial subgroup is in $\mathcal{D}$, the space $E_{\mathcal{D}}(G)$ is contractible. Restricting the $G$-action to the subgroup $\pi \times 1 \subseteq G$ gives a free $\pi$-action, since $E_{\mathcal{D}}(G)$ has isotropy in $\mathcal{D}$ and $(\pi \times 1) \cap H$ is trivial for all $H \in \mathcal{D}$. Hence $E_{\mathcal{D}}(G)$ is a $k$-dimensional model for $E(\pi)$, the classifying space for free $\pi$ actions, and it follows that $\operatorname{cd}(\pi) \leq k$.

The general algebraic result of Proposition 3.5.2 follows from Shapiro's lemma in Bredon cohomology [19, Proposition 3.31], which gives isomorphisms

$$
H^{*}(\pi ; M) \cong H_{\mathcal{D}}^{*}\left(G ; \operatorname{coind}_{I}(M)\right)
$$

for each $\pi$-module $M$. Here the co-induction is along the inclusion functor $I: \mathcal{O}_{\{1\}}(\pi \times 1) \rightarrow$ $\mathcal{O}_{\mathcal{D}}(G)$. This argument does not require the assumption $k \geq 3$.

Corollary 3.5.3. Suppose that $\pi=\mathbb{Z}^{k}$. Then $\operatorname{cd}_{\mathcal{D}}(\pi \times \pi)=\operatorname{cd}(\pi)=\operatorname{TC}(\pi)=k$.

Proof. The space $\mathbb{R}^{k}$ is a free, contractible $\pi$-CW-complex where the action is given by $(a, x) \mapsto a+x$ for $a \in \mathbb{Z}^{k}$ and $x \in \mathbb{R}^{k}$. We may promote this $\pi$-action to a $G$-action on $\mathbb{R}^{k}$, by setting $((a, b), x) \mapsto a-b+x$ (here we use the assumption that $\pi$ is abelian). It is easily seen that $\mathbb{R}^{k}$ with this $G$-action becomes a model for $E_{\mathcal{D}}(G)$. The inequality $\operatorname{cd}_{\mathcal{D}}(\pi \times \pi) \leq \operatorname{cd}(\pi)=k$ is now immediate and the inverse inequality $\operatorname{cd}(\pi) \leq \operatorname{cd}_{\mathcal{D}}(\pi \times \pi)$ is Proposition 3.5.2. The equality $\mathrm{TC}\left(\mathbb{Z}^{k}\right)=\mathrm{TC}\left(T^{k}\right)=k$ is well-known, see [11].

Corollary 3.5.4. Let $X$ be a finite aspherical complex with fundamental group $\pi=$ $\pi_{1}\left(X, x_{0}\right)$, and let $K \leq G=\pi \times \pi$ be a subgroup such that $K \cap H=\{1\}$ for all $H \in \mathcal{D}$. Then $\operatorname{cd}(K) \leq \mathrm{TC}(X)$.

Proof. Under these assumptions any model for $E_{\mathcal{D}}(G)$ is free and contractible when viewed as a $K$-CW-complex, hence it is a model for $E(K)$. The same is true for $E(G)$. Letting $k:=\mathrm{TC}(X)$, we get a sequence of $G$-maps $E(G) \rightarrow L \rightarrow E_{\mathcal{D}}(G)$, where $L$ is a $G$-CW complex of dimension $k$. Restricting to $K$-actions we get, up to $K$-homotopy, a factorisation 
of the identity map $E(K) \rightarrow L \rightarrow E(K)$. This obviously implies that any cohomology class in $H^{m}(K, M)$ with $m>k$ vanishes, i.e. $\operatorname{cd}(K) \leq k$, as stated.

As a particular case of the above, let $K=A \times B$ where $A$ and $B$ are subgroups of $\pi$ such that $g A g^{-1} \cap B=\{1\}$ for all $g \in \pi$. We obtain that $\operatorname{cd}(A \times B) \leq \mathrm{TC}(\pi)$, which recovers the main result of $[21]$.

\section{Lower bounds FOR TC $(X)$ VIA Bredon COHOMOLOGY}

In this section we shall give lower bounds for the topological complexity using Bredon cohomology.

First we recall the basic constructions.

4.1. The family $\mathcal{D}$. Let $\pi$ be a discrete group, we shall denote $G=\pi \times \pi$. As above, we denote by $\mathcal{D}$ the smallest family of subgroups $H \subset \pi \times \pi=G$ which contains the diagonal $\Delta \subset \pi \times \pi$, the trivial subgroup and which is closed under taking conjugations and finite intersections. It is easy to see that a nontrivial subgroup $H \subset \pi \times \pi$ belongs to $\mathcal{D}$ iff it is of the form

$$
H_{b, S}=\left\{\left(a, b a b^{-1}\right), a \in Z(S)\right\},
$$

where $b \in \pi$ and $Z(S)$ denotes the centraliser of a finite set of elements $S \subset \pi$, i.e. $Z(S)=\{a \in \pi, s a=a s$ for any $s \in S\}$.

We denote by $\mathcal{O}_{\mathcal{D}}$ the orbit category with objects transitive left $G$-actions having isotropy in $\mathcal{D}$ and with $G$-equivariant maps as morphisms, see [2]. Objects of the category $\mathcal{O}_{\mathcal{D}}$ have the form $G / H$ where $H \in \mathcal{D}$.

4.2. $\mathcal{O}_{\mathcal{D}}$-modules and their principal components. $A$ (right) $\mathcal{O}_{\mathcal{D}}$-module $\underline{M}$ is a contravariant functor on the category of orbits $\mathcal{O}_{\mathcal{D}}$ with values in the category of abelian groups. Such a module is determined by the abelian groups $\underline{M}(G / H)$ where $H \in \mathcal{D}$, and by a group homomorphism

$$
\underline{M}(G / H) \rightarrow \underline{M}\left(G / H^{\prime}\right)
$$

associated with any $G$-equivariant map $G / H^{\prime} \rightarrow G / H$ satisfying the usual compatibility conditions, expressing the fact that $\underline{M}$ is a functor.

The abelian group $M=\underline{M}(G / 1)$ is a left $\mathbb{Z}[\pi \times \pi]$-module; an element $(g, h) \in \pi \times \pi$ acts on $\pi \times \pi$ by right translation and applying the functor $\underline{M}$ this defines an action on $M$. We shall call the $\mathbb{Z}[\pi \times \pi]$-module $M$ the principal component of $\underline{M}$.

Example 4.2.1. Let $X$ be a left $G$-set. One defines an $\mathcal{O}_{\mathcal{D}}$-module $\underline{M}_{X}$ by

$$
\underline{M}_{X}(?)=\mathbb{Z}[?, X]_{G}
$$

In other words, $\underline{M}_{X}(G / H)$ is the free abelian group generated by the set of $G$-equivariant maps

$$
[G / H, X]_{G}=X^{H}
$$

The homomorphism associated to a morphism $f: G / H^{\prime} \rightarrow G / H$ is the map $X^{H} \rightarrow X^{H^{\prime}}$ given by $x \mapsto f(1,1) \cdot x \in X^{H^{\prime}}$ for $x \in X^{H}$. 
If the set $X$ is such that the isotropy subgroup of any point $x \in X$ belongs to the family $\mathcal{D}$ then the $\mathcal{O}_{\mathcal{D}}$-module $\underline{M}_{X}$ is free and hence projective, see [35], chapter 1 or [28], chapter 2 .

The principal component of the $\mathcal{O}_{\mathcal{D}}$-module $\underline{M}_{X}$ is $M=\mathbb{Z}[X]$, the free abelian group generated by $X$. The left action of $G$ on $\mathbb{Z}[X]$ is induced by the left action of $G$ on $X$.

Any equivariant map between $G$-sets $f: X \rightarrow Y$ induces naturally a homomorphism of the $\mathcal{O}_{\mathcal{D}}$-modules $f_{*}: \underline{M}_{X} \rightarrow \underline{M}_{Y}$.

Next we consider a few special cases of the previous example.

Example 4.2.2. Taking $X=*$, the one point orbit, we obtain the module $\underline{M}_{X}$ which will be denoted $\underline{\mathbb{Z}}$. It associates $\mathbb{Z}$ to any orbit $\pi \times \pi / H$ with the identity homomorphism associated to any morphism of the orbit category $\mathcal{O}_{\mathcal{D}}$. Note that $\underline{\mathbb{Z}}$ is not a free $\mathcal{O}_{\mathcal{D}}$-module since $G=\pi \times \pi$ is not in $\mathcal{D}$.

Example 4.2.3. In Example 4.2.1 take $X=\pi$, the group $\pi$ viewed as a $G=\pi \times \pi$ set via the action $(x, y) \cdot g=x g y^{-1}$. The isotropy subgroup of an element $g \in \pi$ is $\left\{\left(x, g^{-1} x g\right), x \in \pi\right\}$ which belongs to the family $\mathcal{D}$ and hence the Bredon module $\underline{M}_{\pi}$ is free. Note that $\underline{M}_{\pi}$ associates the abelian group $\mathbb{Z}\left[\pi^{H}\right]$ to any orbit $G / H$.

If $H=H_{b, S}$ then $\pi^{H}$ coincides with $Z(Z(S)) \cdot b^{-1}$. In general, $\pi^{H}$ is not a subgroup.

Example 4.2.4. This is a generalisation of the previous example. For an integer $s \geq 1$, consider the $s$-th Cartesian power $\pi^{s}$ as a $G=\pi \times \pi$-set via the action $(x, y) \cdot\left(g_{1}, \ldots, g_{s}\right)=$ $\left(x g_{1} y^{-1}, \ldots, x g_{s} y^{-1}\right)$. The isotropy subgroup of an element $\left(g_{1}, \ldots, g_{s}\right)$ is the intersection of the isotropy subgroups of $g_{i}$ for $i=1, \ldots, s$, hence it can be presented as $H_{b, S}$ with $b=g_{1}$ and $S=\left\{g_{1} g_{2}^{-1}, g_{1} g_{3}^{-1}, \ldots, g_{1} g_{s}^{-1}\right\}$. We obtain a free Bredon module $\underline{M}_{\pi^{s}}, s \geq 1$. Its principal component is the module $\mathbb{Z}\left[\pi^{S}\right]$.

4.3. Bredon cohomology. Now we recall the construction of Bredon cohomology, see for example [31].

Let $X$ be a $G$-CW-complex such that the isotropy subgroup of every point $x \in X$ belongs to the family $\mathcal{D}$. For every subgroup $H \in \mathcal{D}$ we may consider the cell complex $X^{H}$ of $H$ fixed points and its cellular chain complex $C_{*}\left(X^{H}\right)$. A $G$-map $\phi: G / K \rightarrow G / L$, where $K, L \in \mathcal{D}$, induces a cellular map $X^{L} \rightarrow X^{K}$ by mapping $x \in X^{L}$ to $g x \in X^{K}$ where $g$ is determined by the equation $\phi(K)=g L$ (thus $g^{-1} K g x=x$ since $g^{-1} K g \subset L$ and therefore $K g x=g x$, i.e. $\left.g x \in X^{K}\right)$. Thus we see that the chain complexes $C_{*}\left(X^{H}\right)$, considered for all $H \in \mathcal{D}$, form a chain complex of right $\mathcal{O}_{\mathcal{D}}$-modules which will be denoted $\underline{C}_{*}(X)$; here $\underline{C}_{*}(X)(G / H)=C_{*}\left(X^{H}\right)$. The principal component of the $\mathcal{O}_{\mathcal{D}^{-c h a i n}}$ complex $\underline{C}_{*}(X)$ is the chain complex $C_{*}(X)$ of left $\mathbb{Z}[G]$-modules.

Note that the complex $\underline{C}_{*}(X)$ is free as a complex of $\mathcal{O}_{\mathcal{D}}$-modules although the complex $C_{*}(X)$ might not be free as a complex of $\mathbb{Z}[G]$-modules.

There is an obvious augmentation $\epsilon: \underline{C}_{0}(X) \rightarrow \underline{\mathbb{Z}}$ which reduces to the usual augmentation $C_{0}\left(X^{H}\right) \rightarrow \mathbb{Z}$ on each subgroup $H \in \mathcal{D}$.

If $\underline{M}$ is a right $\mathcal{O}_{\mathcal{D}}$-module, we may consider the cochain complex of $\mathcal{O}_{\mathcal{D}}$-morphisms $\operatorname{Hom}_{\mathcal{O}_{\mathcal{D}}}\left(\underline{C}_{*}(X), \underline{M}\right)$. Its cohomology

$$
H_{\mathcal{D}}^{*}(X ; \underline{M})=H^{*}\left(\operatorname{Hom}_{\mathcal{O}_{\mathcal{D}}}\left(\underline{C}_{*}(X) ; \underline{M}\right)\right)
$$


is the Bredon equivariant cohomology of $X$ with coefficients in $\underline{M}$.

Let $M$ denote the principal component of $\underline{M}$. By reducing to the principal components we obtain a homomorphism of cochain complexes

$$
\operatorname{Hom}_{\mathcal{O}_{\mathcal{D}}}\left(\underline{C}_{*}(X), \underline{M}\right) \rightarrow \operatorname{Hom}_{\mathbb{Z}[G]}\left(C_{*}(X), M\right)
$$

and the associated homomorphism on cohomology groups

$$
H_{\mathcal{D}}^{i}(X ; \underline{M}) \rightarrow H_{G}^{i}(X, M) .
$$

4.4. If the action of $G$ on $X$ is free then obviously the homomorphism (13) is an isomorphism and

$$
H_{\mathcal{D}}^{i}(X ; \underline{M}) \cong H^{i}(X / G ; M),
$$

where on the right we have the usual twisted cohomology. In particular we obtain

$$
H_{\mathcal{D}}^{n}(E(\pi \times \pi) ; \underline{M})=H^{n}(\pi \times \pi ; M) .
$$

4.5. Suppose now that $X=E(\pi)$, viewed as a left $G$-CW-complex, where $G=\pi \times \pi$, see $\S 3.4$. We know that $E(\pi)$ is a model for the classifying space $E_{\mathcal{D}}(G)$ (as we established in $\S 3.4)$ and the classifying complex $E_{\mathcal{D}}(G)$ is unique up to $G$-homotopy. Hence we may use the notation

$$
H_{\mathcal{D}}^{*}(E(\pi) ; \underline{M})=H_{\mathcal{D}}^{*}(\pi \times \pi ; \underline{M}) .
$$

We obtain that the number $\operatorname{cd}_{\mathcal{D}}(\pi \times \pi)$ coincides with the minimal integer $n$ such that $H_{\mathcal{D}}^{i}(\pi \times \pi ; \underline{M})=0$ for all $i>n$ and for all $\mathcal{O}_{\mathcal{D}}$-modules $\underline{M}$.

4.6. Consider now the effect of the equivariant map $F: E(\pi \times \pi) \rightarrow E(\pi)$, see (4). Note that any two equivariant maps $E(\pi \times \pi) \rightarrow E(\pi)$ are equivariantly homotopic. The induced map on Bredon cohomology

$$
F^{*}: H_{\mathcal{D}}^{i}(E(\pi) ; \underline{M}) \rightarrow H_{\mathcal{D}}^{i}(E(\pi \times \pi) ; \underline{M})
$$

in the notations introduced in $\S 4.4$ and $\S 4.5$ produces a homomorphism

$$
\Phi: H_{\mathcal{D}}^{i}(\pi \times \pi ; \underline{M}) \rightarrow H^{i}(\pi \times \pi ; M)
$$

which connects the Bredon cohomology with the usual group cohomology.

Now we may state a result which gives useful lower bounds for the topological complexity $\mathrm{TC}(X)$.

Theorem 4.1. Let $X$ be a finite aspherical cell complex with fundamental group $\pi$. Suppose that for some $\mathcal{O}_{\mathcal{D}}$-module $\underline{M}$ there exists a Bredon cohomology class

$$
\underline{\alpha} \in H_{\mathcal{D}}^{n}(\pi \times \pi ; \underline{M})
$$

such that the class

$$
\Phi(\underline{\alpha}) \neq 0 \in H^{n}(\pi \times \pi ; M)
$$

is nonzero. Then $\mathrm{TC}(X) \geq n$. Here $M$ denotes the principal component of $\underline{M}$. 
Proof. Suppose that $\mathrm{TC}(X)<n$. Then by Theorem 3.1 the map $F: E(\pi \times \pi) \rightarrow E(\pi)$ admits a factorisation

$$
E(\pi \times \pi) \rightarrow L \rightarrow E(\pi)
$$

where $L$ is a $G$-CW-complex of dimension less than $n$. Then the homomorphism

$$
\Phi: H_{\mathcal{D}}^{n}(\pi \times \pi ; \underline{M}) \rightarrow H^{n}(\pi \times \pi ; M)
$$

factors as

$$
\Phi: H_{\mathcal{D}}^{n}(\pi \times \pi ; \underline{M}) \rightarrow H_{\mathcal{D}}^{n}(L ; \underline{M}) \rightarrow H^{n}(\pi \times \pi ; M)
$$

and the middle group vanishes since $\operatorname{dim} L<n$. This contradicts our assumption that $\Phi(\underline{\alpha}) \neq 0$ for some $\underline{\alpha} \in H_{\mathcal{D}}^{n}(\pi \times \pi ; \underline{M})$.

Remark 4.6.1. Theorem 4.1 can be compared to the classical result concerning the Lusternik - Schirelman category (see Eilenberg - Ganea [10] or Schwarz [34]) stating that for an aspherical space $X$ the existence of a nonzero cohomology class $H^{n}(X ; M)$ (with some local coefficient system $M)$ implies that $\operatorname{cat}(X) \geq n$. It is not true that $\mathrm{TC}(X) \geq n$ if $H^{n}(X \times X ; M) \neq 0$ for $X$ aspherical. For example, in the case of the circle $X=S^{1}$ we know that $\mathrm{TC}(X)=1$ while $H^{2}(X \times X ; \mathbb{Z}) \neq 0$. Theorem 4.1 imposes a condition on the nontrivial cohomology class in the usual twisted cohomology to be extendable to a class in Bredon cohomology. We will investigate this property further in $\S 7$.

\section{The CANONical Class in BRedon COHOMOlOGY AND its Universality}

In this section we define a special Bredon cohomology class which will play an important role in this paper.

5.1. The canonical class. Consider an $\mathcal{O}_{\mathcal{D}}$-module $M_{X}($ ? $)=\mathbb{Z}[?, X]_{G}$ where $X$ is a $G$ set, see Example 4.2.1. Recall that $G$ denotes the group $\pi \times \pi$. The unique map $X \rightarrow *$ is $G$-invariant and induces a homomorphism of Bredon modules $\epsilon: \underline{M}_{X} \rightarrow \underline{M}_{*}=\underline{\mathbb{Z}}$, called the augmentation. We denote by $\underline{I}_{X}$ the kernel of $\epsilon$. Clearly, $\underline{I}_{X}$ is a Bredon module whose value on an orbit $G / H$ is

$$
\underline{I}_{X}(G / H)=\operatorname{ker}\left[\epsilon: \mathbb{Z}\left[X^{H}\right] \rightarrow \mathbb{Z}\right] .
$$

As a special case of the previous construction we obtain the Bredon module $\underline{I}_{\pi}$ (where $X=\pi$, as in Example 4.2.3). Here

$$
\underline{I}_{\pi}(G / H)=\operatorname{ker}\left[\epsilon: \mathbb{Z}\left[\pi^{H}\right] \rightarrow \mathbb{Z}\right] \equiv I\left(\pi^{H}\right) .
$$

We shall shorten the notation $\underline{I}_{\pi}$ to $\underline{I}$. The principal component of $\underline{I}$ is the augmentation ideal $I=\operatorname{ker}[\epsilon: \mathbb{Z}[\pi] \rightarrow \mathbb{Z}]$.

One obtains a short exact sequence of Bredon modules

$$
0 \rightarrow \underline{I} \rightarrow \underline{M}_{\pi} \stackrel{\epsilon}{\rightarrow} \underline{\mathbb{Z}} \rightarrow 0 .
$$

The latter defines a Bredon cohomology class

$$
\mathfrak{u} \in \operatorname{Ext}_{\mathcal{O}_{\mathcal{D}}}^{1}(\underline{\mathbb{Z}}, \underline{I}) \equiv H_{\mathcal{D}}^{1}(\pi \times \pi ; \underline{I}) .
$$


We shall call $\mathfrak{u}$ the canonical class in Bredon cohomology. It is a refinement of the ordinary canonical class

$$
\mathfrak{v} \in H^{1}(\pi \times \pi ; I)
$$

which was defined in [6]. In [17], $\S 3$ it is shown that $\mathfrak{v}$ coincides with the class represented by the principal components of the sequence (16), i.e. by the exact sequence of left $\mathbb{Z}[\pi \times \pi]$ modules

$$
0 \rightarrow I \rightarrow \mathbb{Z}[\pi] \rightarrow \mathbb{Z} \rightarrow 0
$$

Hence, the principal component of the class $\mathfrak{u}$ (i.e. the image of $\mathfrak{u}$ under the homomorphism $(14)$ ), coincides with $\mathfrak{v}$.

The canonical class $\mathfrak{v}$ is closely related to the Berstein - Schwarz class

$$
\mathfrak{b} \in H^{1}(\pi ; I)
$$

which is represented by the exact sequence (17) viewed as a sequence of left $\mathbb{Z}[\pi]$-modules.

5.2. The classes $\mathfrak{u}^{n}$. Next we define classes

$$
\mathfrak{u}^{n} \in H_{\mathcal{D}}^{n}\left(\pi \times \pi ; \underline{I}^{n}\right), \quad n=1,2, \ldots
$$

In this paper we shall treat these classes formally and call them the powers of the canonical class $\mathfrak{u}$ without trying to justify this name. However we shall show that the principal component of the class $\mathfrak{u}^{n}$ is the $n$-fold cup product $\mathfrak{v} \cup \mathfrak{v} \cup \cdots \cup \mathfrak{v}=\mathfrak{v}^{n}$ of the canonical class $\mathfrak{v} \in H^{1}(\pi \times \pi ; I)$.

The Bredon module $\underline{I}^{n}$ is defined by

$$
\underline{I}^{n}(G / H)=I\left(\pi^{H}\right) \otimes_{\mathbb{Z}} I\left(\pi^{H}\right) \otimes_{\mathbb{Z}} \cdots \otimes_{\mathbb{Z}} I\left(\pi^{H}\right), \quad H \in \mathcal{D} .
$$

We shall define the class $\mathfrak{u}^{n}$ by describing an explicit exact sequence of $\mathcal{O}_{\mathcal{D}}$-modules

$$
0 \rightarrow \underline{I}^{n} \rightarrow \underline{C}_{n-1} \stackrel{d}{\rightarrow} \underline{C}_{n-2} \stackrel{d}{\rightarrow} \cdots \stackrel{d}{\rightarrow} \underline{C}_{0} \rightarrow \underline{\mathbb{Z}} \rightarrow 0
$$

in which the intermediate $\mathcal{O}_{\mathcal{D}}$-modules $\underline{C}_{0}, \underline{C}_{1}, \ldots, \underline{C}_{n-1}$ are projective. If

$$
\underline{P}_{*}: \quad \cdots \underline{P}_{2} \rightarrow \underline{P}_{1} \rightarrow \underline{P}_{0} \rightarrow \underline{\mathbb{Z}} \rightarrow 0
$$

is an $\mathcal{O}_{\mathcal{D}}$-projective resolution of $\underline{\mathbb{Z}}$, we obtain a commutative diagram (unique up to chain homotopy)

$$
\begin{array}{ccccccccccccc}
\underline{P}_{n+1} & \rightarrow & \underline{P}_{n} & \rightarrow & \underline{P}_{n-1} & \rightarrow & \cdots & \underline{P}_{0} & \rightarrow & \underline{\mathbb{Z}} & \rightarrow & 0 \\
\downarrow & & \downarrow f & \downarrow & & & \downarrow & & \downarrow & & \downarrow & & \\
0 & \rightarrow & \underline{I}^{n} & \rightarrow & \underline{C}_{n-1} & \rightarrow & \cdots & \underline{C}_{0} & \rightarrow & \underline{\mathbb{Z}} & \rightarrow & 0 .
\end{array}
$$

The $\mathcal{O}_{\mathcal{D}}$-homomorphism $f$ is a cocycle, and its cohomology class

$$
\{f\} \in H^{n}\left(\operatorname{Hom}_{\mathcal{O}_{\mathcal{D}}}\left(\underline{P}_{*}, \underline{I}^{n}\right)\right)=H_{\mathcal{D}}^{n}\left(\pi \times \pi ; \underline{I}^{n}\right)
$$

is independent of the choice of the chain map represented by the diagram above. We define the $n$-th power of the canonical class $\mathfrak{u}^{n}$ as the cohomology class $\{f\}$. 
The principal components of the exact sequence (18) define an exact sequence of left $\mathbb{Z}[\pi \times \pi]=\mathbb{Z}[G]$-modules

$$
0 \rightarrow \underline{I}^{n}(G / 1)=I^{n} \rightarrow \underline{C}_{n-1}(G / 1) \stackrel{d}{\rightarrow} \underline{C}_{n-2}(G / 1) \stackrel{d}{\rightarrow} \cdots \stackrel{d}{\rightarrow} \underline{C}_{0}(G / 1) \rightarrow \mathbb{Z} \rightarrow 0 .
$$

This sequence determines a class in

$$
\operatorname{Ext}_{\mathbb{Z}[\pi \times \pi]}^{n}\left(\mathbb{Z}, I^{n}\right)=H^{n}\left(\pi \times \pi ; I^{n}\right)
$$

which is the principal component of the class $\mathfrak{u}^{n}$. We shall identify the principal component of $\mathfrak{u}^{n}$ with $\mathfrak{v}^{n}$, see Theorem 5.2.

5.3. Construction of the complex (18). Here we shall generalise a construction of Dranishnikov and Rudyak [8]; see also [17].

We shall use the operation $\otimes_{\mathbb{Z}}$ of tensor product of $\mathcal{O}_{\mathcal{D}}$-modules which is defined as follows. For two right $\mathcal{O}_{\mathcal{D}}$-modules $\underline{M}$ and $\underline{N}$ we define $\underline{M} \otimes_{\mathbb{Z}} \underline{N}$ by the formula

$$
\left(\underline{M} \otimes_{\mathbb{Z}} \underline{N}\right)(G / H)=\underline{M}(G / H) \otimes_{\mathbb{Z}} \underline{N}(G / H), \quad H \in \mathcal{D},
$$

with the obvious action on morphisms.

The following obvious remark will be used in the sequel. Suppose that

$$
0 \rightarrow \underline{M}_{1} \rightarrow \underline{M}_{2} \rightarrow \underline{M}_{3} \rightarrow 0
$$

is an exact sequence of right $\mathcal{O}_{\mathcal{D}}$-modules and let $\underline{N}$ be a right $\mathcal{O}_{\mathcal{D}}$-module such that for any $H \in \mathcal{D}$ the module $\underline{N}(G / H)$ is free as an abelian group. Then the sequence

$$
0 \rightarrow \underline{N} \otimes_{\mathbb{Z}} \underline{M}_{1} \rightarrow \underline{N} \otimes_{\mathbb{Z}} \underline{M}_{2} \rightarrow \underline{N} \otimes_{\mathbb{Z}} \underline{M}_{3} \rightarrow 0
$$

is also exact.

Let $X$ and $Y$ be left $G$-sets, where $G=\pi \times \pi$. Consider the $\mathcal{O}_{\mathcal{D}}$-modules $\underline{M}_{X}$ and $\underline{M}_{Y}$, see Example 4.2.1. Note that the tensor product $\underline{M}_{X} \otimes_{\mathbb{Z}} \underline{M}_{Y}$ can be naturally identified with $\underline{M}_{X \times Y}$. We know that the modules $\underline{M}_{X}, \underline{M}_{Y}$ and $\underline{M}_{X \times Y}$ are free iff the isotropy subgroups of all elements of $X$ and $Y$ belong to $\mathcal{D}$.

Tensoring the short exact sequence

$$
0 \rightarrow \underline{I}_{Y} \rightarrow \underline{M}_{Y} \stackrel{\epsilon}{\rightarrow} \underline{\mathbb{Z}} \rightarrow 0
$$

with $\underline{M}_{X}$ we obtain an exact sequence

$$
0 \rightarrow \underline{M}_{X} \otimes_{\mathbb{Z}} \underline{I}_{Y} \rightarrow \underline{M}_{X \times Y} \rightarrow \underline{M}_{X} \rightarrow 0
$$

in which $\underline{M}_{X}$ and $\underline{M}_{X \times Y}$ are free and hence the sequence (20) splits. We conclude: If the isotropy subgroups of all elements of $X \sqcup Y$ belong to $\mathcal{D}$, then the $\mathcal{O}_{\mathcal{D}}$-module $\underline{M}_{X} \otimes_{\mathbb{Z}} \underline{I}_{Y}$ is projective.

Next we prove by induction that for any $r \geq 0$ and for any left $G$-set $X$ such that the isotropy subgroup of any element of $X$ lies in the family $\mathcal{D}$, the $\mathcal{O}_{\mathcal{D}}$-module $\underline{M}_{X} \otimes_{\mathbb{Z}} \underline{I}^{r}$ is projective. Here $\underline{I}^{r}$ denotes the $r$-fold tensor product $\underline{I} \otimes_{\mathbb{Z}} \underline{I} \otimes_{\mathbb{Z}} \cdots \otimes_{\mathbb{Z}} \underline{I}$ and, as above, $\underline{I}$ denotes $\underline{I}_{\pi}$. For $r=0$ our statement is obvious since under our assumptions on $X$ the $\mathcal{O}_{\mathcal{D}}$-module $\underline{M}_{X}$, is free and hence projective. For $r=1$ this statement follows from 
the italicised claim of the previous paragraph taken with $Y=\pi$. To perform the step of induction for $r \geq 2$ we tensor the exact sequence (19) by $\underline{I}^{r-1}$ obtaining the exact sequence

$$
0 \rightarrow \underline{M}_{X} \otimes_{\mathbb{Z}} \underline{I}^{r} \rightarrow \underline{M}_{(X \times \pi)} \otimes_{\mathbb{Z}} \underline{I}^{r-1} \rightarrow \underline{M}_{X} \otimes_{\mathbb{Z}} \underline{I}^{r-1} \rightarrow 0
$$

in which the middle and the right $\mathcal{O}_{\mathcal{D}}$-modules are projective (by the induction hypothesis) implying that $\underline{M}_{X} \otimes_{\mathbb{Z}} \underline{I}^{r}$ is projective as well.

Starting from the short exact sequence (16) and tensoring with $\underline{I}$ we iteratively obtain short exact sequences of $\mathcal{O}_{\mathcal{D}}$-modules

$$
0 \rightarrow \underline{I}^{r} \stackrel{i \otimes 1}{\rightarrow} \underline{M}_{\pi} \otimes_{\mathbb{Z}} \underline{I}^{r-1} \stackrel{\epsilon \otimes 1}{\rightarrow} \underline{I}^{r-1} \rightarrow 0, \quad r=1,2, \ldots
$$

Splicing them for $r=1,2, \ldots, n$ we obtain the long exact sequence of $\mathcal{O}_{\mathcal{D}}$-modules

(22) $0 \rightarrow \underline{I}^{n} \rightarrow \underline{M}_{\pi} \otimes_{\mathbb{Z}} \underline{I}^{n-1} \rightarrow \underline{M}_{\pi} \otimes_{\mathbb{Z}} \underline{I}^{n-2} \rightarrow \cdots \rightarrow \underline{M}_{\pi} \otimes_{\mathbb{Z}} \underline{I} \rightarrow \underline{M}_{\pi} \rightarrow \underline{\mathbb{Z}} \rightarrow 0$.

This is a version of the complex (18). Naturally, there exist many other chain complexes representing the same cohomology class $\mathfrak{u}^{n}$.

5.4. Universality of the canonical class. In this subsection we prove the following statement which is a generalisation of the well-known result of A.S. Schwarz (see [34], Proposition 34).

Theorem 5.1. For any $\mathcal{O}_{\mathcal{D}}$-module $\underline{M}$ and for any cohomology class

$$
\underline{\alpha} \in H_{\mathcal{D}}^{n}(\pi \times \pi ; \underline{M})
$$

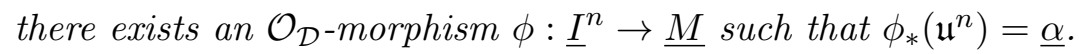

Proof. One may construct a projective $\mathcal{O}_{\mathcal{D}}$-resolution of $\underline{\mathbb{Z}}$ extending (18)

$$
\cdots \stackrel{d}{\rightarrow} \underline{C}_{n} \stackrel{d}{\rightarrow} \underline{C}_{n-1} \stackrel{d}{\rightarrow} \underline{C}_{n-2} \stackrel{d}{\rightarrow} \cdots \stackrel{d}{\rightarrow} \underline{C}_{0} \rightarrow \underline{\mathbb{Z}} \rightarrow 0 .
$$

The class $\underline{\alpha}$ can be viewed as a cohomology class of the cochain complex $\operatorname{Hom}_{\mathcal{O}_{\mathcal{D}}}\left(\underline{C}_{*}, \underline{M}\right)$. Let $f: \underline{C}_{n} \rightarrow \underline{M}$ be a cocycle representing $\alpha$. In the diagram

$$
\begin{aligned}
& \underline{C}_{n+1} \stackrel{\stackrel{d}{\rightarrow}}{\underline{C}_{n}} \stackrel{\stackrel{d}{\rightarrow}}{\underline{I}^{n}} \stackrel{\stackrel{d}{\rightarrow}}{\rightarrow} 0 \\
& \downarrow f \swarrow \phi
\end{aligned}
$$

\section{$\underline{M}$}

the row is exact and the existence of a $\mathcal{O}_{\mathcal{D}}$-homomorphism $\phi: \underline{I}^{n} \rightarrow \underline{M}$ follows from the assumption that $f$ is a cocycle. We claim that $\phi_{*}\left(\mathfrak{u}^{n}\right)=\underline{\alpha}$. Indeed, the class $\mathfrak{u}^{n}$ is represented by a similar diagram

$$
\begin{aligned}
\underline{C}_{n+1} \stackrel{d}{\rightarrow} & \underline{C}_{n} \quad \stackrel{d}{\rightarrow} \quad \underline{I}^{n} \quad \stackrel{d}{\rightarrow} 0 \\
& \downarrow g \quad \swarrow \text { id } \\
& \underline{I}^{n}
\end{aligned}
$$


implying that $\phi \circ g=f$. Hence we see that the cocycle representing the class $\underline{\alpha}$ is obtained from the cocycle representing $\mathfrak{u}^{n}$ by composing with $\phi$.

Theorem 5.1 obviously implies:

Corollary 5.4.1. One has

$$
\operatorname{cd}_{\mathcal{D}}(\pi \times \pi)=\operatorname{height}(\mathfrak{u}),
$$

where the integer height $(\mathfrak{u})$ is defined as the largest $n$ such that the class $\mathfrak{u}^{n} \in H_{\mathcal{D}}^{n}\left(\pi \times \pi ; \underline{I}^{n}\right)$ is nonzero.

Theorem 5.2. For any integer $n \geq 1$ the image of the class

$$
\mathfrak{u}^{n} \in H_{\mathcal{D}}^{n}\left(\pi \times \pi ; \underline{I}^{n}\right)
$$

under the homomorphism (14) coincides with the n-fold cup-power

$$
\Phi\left(\mathfrak{u}^{n}\right)=\mathfrak{v}^{n}=\mathfrak{v} \cup \mathfrak{v} \cup \cdots \cup \mathfrak{v} \in H^{n}\left(\pi \times \pi ; I^{n}\right)
$$

of the canonical class $\mathfrak{v} \in H^{1}(\pi \times \pi ; I)$.

Proof. The principal components of the complex (22) is exactly the chain complex (12) from [17] and our statement is identical to Lemma 3.1 from [17].

\section{Principal $\mathcal{O}_{\mathcal{D}}$-MODUleS}

In this section $G$ denotes the group $\pi \times \pi$ and $\mathcal{D}$ is the family of subgroups of $G$ defined in Example 3.3.1, see also $§ 4.1$.

6.1. Let $\underline{M}$ be an $\mathcal{O}_{\mathcal{D}}$-module. The principal component of $\underline{M}$ is defined as $\underline{M}(G / 1)=A$ which (as we noted in $\S 4.2$ ) has the structure of a left $\mathbb{Z}[G]$-module. Note that for any orbit $G / H$ we have an $\mathcal{O}_{\mathcal{D}}$-morphism $f_{H}: G \rightarrow G / H$ given by $g \mapsto g H$ which induces a homomorphism

$$
\underline{M}\left(f_{H}\right): \underline{M}(G / H) \rightarrow \underline{M}(G / 1)=A .
$$

For $a \in H$ we have $f_{H}=f_{H} \circ r_{a}$ where $r_{a}: G \rightarrow G$ is the right multiplication by $a$, i.e. $r_{a}(g)=g a$. Applying the functor $\underline{M}$ we see that the homomorphism $\phi_{H} \equiv \underline{M}\left(f_{H}\right)$ takes values in $A^{H}$, i.e.

$$
\phi_{H}: \underline{M}(G / H) \rightarrow A^{H} .
$$

Definition 6.1.1. We shall say that an $\mathcal{O}_{\mathcal{D}}$-module $\underline{M}$ is principal if for any subgroup $H \in \mathcal{D}$ the homomorphism

$$
\phi_{H}=\underline{M}\left(f_{H}\right): \underline{M}(G / H) \rightarrow A^{H}
$$

is an isomorphism. 
Let $\underline{M}$ be a principal $\mathcal{O}_{\mathcal{D}}$-module. Let $H, K \in \mathcal{D}$ and let $a \in G$ be such that $a^{-1} H a \subset K$. Then we have an $\mathcal{O}_{\mathcal{D}}$-morphism $f_{a}: G / H \rightarrow G / K$ where $f_{a}(g H)=g a K$ for any $g \in G$. We obtain the commutative diagram

$\begin{array}{ccc}G & \stackrel{r_{a}}{\rightarrow} & G \\ f_{H} \downarrow & & \downarrow f_{K} \\ G / H & \stackrel{f_{a}}{\rightarrow} & G / K .\end{array}$

of orbits and applying the functor $\underline{M}$ we obtain the commutative diagram

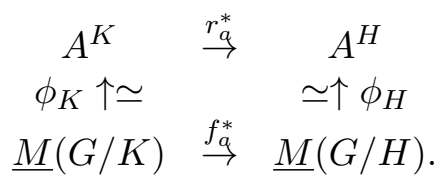

where $r_{a}^{*}$ is multiplication by $a$. Thus we see that the structure of a principal $\mathcal{O}_{\mathcal{D}}$-module $\underline{M}$ is fully determined by the left $\mathbb{Z}[G]$-module $A$ (the principal component of $\underline{M}$ ). Viewing $A$ as a left $G$-set we may write

$$
\underline{M}(G / H)=[G / H, A]=A^{H} .
$$

Principal modules appear in the book of G. Bredon [2] as Example (2), page I-10.

6.2. As an example consider the $\mathcal{O}_{\mathcal{D}}$-module $\underline{M}_{X}(?)=\mathbb{Z}[?, X]_{G}$ (see Example 4.2.1) where $X$ is a left $G$-set. In this case the principal component is $\mathbb{Z}[X]$ viewed as a left $\mathbb{Z}[G]$-module. For an orbit $G / H$ with $H \in \mathcal{D}$ we have $\underline{M}_{X}(G / H)=\mathbb{Z}\left[X^{H}\right]$ and the map $f_{H}: G / 1 \rightarrow G / H$ induces a homomorphism

$$
\mathbb{Z}\left[X^{H}\right] \rightarrow(\mathbb{Z}[X])^{H}
$$

which in general is an inclusion.

Lemma 6.2.1. The homomorphism (25) is an isomorphism if and only if for any $H \in \mathcal{D}$, the set $X$ viewed as an $H$-set, has the following property: any orbit of $H$ contained in $X$ is either infinite or a single point.

Proof. Suppose that $X$ satisfies the condition of the Lemma. For $H \in \mathcal{D}$ we may split $X$ into a disjoint union of $H$-orbits $X=\sqcup_{j} X_{j}$ where each $X_{j}$ is either a single point or infinite. Then $\mathbb{Z}[X]=\oplus_{j} \mathbb{Z}\left[X_{j}\right]$ and $\mathbb{Z}[X]^{H}=\oplus_{j} \mathbb{Z}\left[X_{j}\right]^{H}$ with $\mathbb{Z}\left[X_{j}\right]^{H}=\mathbb{Z}\left[X_{j}\right]$ if $X_{j}$ is a single point and $\mathbb{Z}\left[X_{j}\right]^{H}=0$ if $X_{j}$ is infinite. On the other hand the set $X^{H}$ is the union of the sets $X_{j}$ which are single points. Hence (25) is an isomorphism.

The inverse statement follows similarly. Namely, suppose that $X_{j} \subset X$ is a finite $G$-orbit which is not a single point. Then the element

$$
\sum_{x \in X_{j}} x \in \mathbb{Z}[X]
$$

is invariant with respect to $H$, i.e. it lies in $(\mathbb{Z}[X])^{H}$ but not in $\mathbb{Z}\left[X^{H}\right]$. 
We want to restate Lemma 6.2.1 in terms of the isotropy subgroups of points of $X$. For a point $x \in X$ denote by $I(x) \subset \pi \times \pi$ its isotropy subgroup. For a subgroup $H \subset G=\pi \times \pi$ one has $x \in X^{H}$ iff $H \subset I(x)$. The orbit of $x$ with respect to $H$ is finite iff $H$ contains $I(x) \cap H$ as a finite index subgroup. Thus we obtain the following Corollary:

Corollary 6.2.2. The $\mathcal{O}_{\mathcal{D}}$-module $\underline{M}_{X}$ is principal if and only if for any $x \in X$ and any subgroup $H \in \mathcal{D}$ the index $[H: H \cap I(x)]$ is either 1 or $\infty$.

For free $\mathcal{O}_{\mathcal{D}}$-modules $\underline{M}_{X}$ the set $X$ has all isotropy subgroups in $\mathcal{D}$. This leads to the following Corollary:

Corollary 6.2.3. Suppose that for any two subgroups $H, H^{\prime} \in \mathcal{D}$ the index $\left[H: H \cap H^{\prime}\right]$ is either 1 or $\infty$. Then any free $\mathcal{O}_{\mathcal{D}}$-module is principal.

Note that the property of the family of subgroups $\mathcal{D}$ described in Corollary 6.2.3 is in fact a property of the group $\pi$ since the family $\mathcal{D}$ depends on the group $\pi$ alone.

Definition 6.2.4. We shall say that a group $\pi$ is principal if any of the following equivalent conditions is satisfied:

(a) Any free $\mathcal{O}_{\mathcal{D}}$-module is principal,

(b) For any two subgroups $H, H^{\prime} \in \mathcal{D}$, the index $\left[H: H \cap H^{\prime}\right]$ is either 1 or infinity,

(c) For any two finite subsets $S, S^{\prime} \subset \pi$ the group $Z(S) / Z\left(S \cup S^{\prime}\right)$ is either infinite or trivial.

Recall that the symbol $Z(S)$ denotes the centraliser of $S$, i.e. the set of all elements $g \in \pi$ which commute with every element of $S$. The equivalence between (a) and (b) follows from Corollaries 6.2.2 and 6.2.3. The equivalence $(b) \sim(c)$ follows from the structure of the groups $H \in \mathcal{D}$.

Example 6.2.5. Let $\pi=\mathbb{Z}^{n}$. Then the class $\mathcal{D}$ contains only two subgroups, the trivial subgroup and the diagonal $\Delta$. The condition of Corollary 6.2 .3 is clearly satisfied, i.e. $\mathbb{Z}^{n}$ is a principal group.

Other examples of principal groups will be described in $\S 8$.

Lemma 6.2.6. Let $0 \rightarrow \underline{M}_{1} \stackrel{\alpha}{\rightarrow} \underline{M}_{2} \stackrel{\beta}{\rightarrow} \underline{M}_{3}$ be an exact sequence of $\mathcal{O}_{\mathcal{D}}$-modules such that the modules $\underline{M}_{2}$ and $\underline{M}_{3}$ are principal. Then the module $\underline{M}_{1}$ is also principal.

Proof. Denote $G=\pi \times \pi$ for short. For any $H \in \mathcal{D}$ we have the following commutative diagram

$$
\begin{aligned}
& 0 \rightarrow M_{1}(G / H) \stackrel{\alpha}{\rightarrow} M_{2}(G / H) \stackrel{\beta}{\rightarrow} M_{3}(G / H) \\
& \downarrow \phi_{H}^{1} \quad \downarrow \phi_{H}^{2} \quad \downarrow \phi_{H}^{3} \\
& 0 \rightarrow \quad A_{1}^{H} \quad \stackrel{\alpha}{\rightarrow} \quad A_{2}^{H} \quad \stackrel{\beta}{\rightarrow} \quad A_{3}^{H}
\end{aligned}
$$

The rows are exact and $\phi_{H}^{2}$ and $\phi_{H}^{3}$ are isomorphisms. By the 5-lemma we obtain that $\phi_{H}^{1}$ is also an isomorphism. Hence $\underline{M}_{1}$ is principal. 
Lemma 6.2 .6 can also be stated as saying that the kernel of a $\mathcal{O}_{\mathcal{D}}$-morphism of principal Bredon modules is principal.

Corollary 6.2.7. Assume that the group $\pi$ is principal. Then the $\mathcal{O}_{\mathcal{D}}$-module $\underline{I}^{n}$ is principal for any $n \geq 1$.

Proof. First let us make the following general remark. Let $X$ and $Y$ be left $\pi \times \pi$-sets with all isotropy subgroups in $\mathcal{D}$. Then the $\mathcal{O}_{\mathcal{D}}$-module $\underline{M}_{X} \otimes_{\mathbb{Z}} \underline{I}_{Y}$ is principal as follows by applying Lemma 6.2.6 to the exact sequence (20) and noting that the free modules $\underline{M}_{X \times Y}$ and $\underline{M}_{X}$ are principal.

The statement of Corollary 6.2.7 now follows by inductively applying the above remark to the exact sequence $(21)$.

Morphisms between principal modules are determined by their effect on the principal components:

Lemma 6.2.8. Let $\underline{M}_{1}$ and $\underline{M}_{2}$ be principal $\mathcal{O}_{\mathcal{D}}$-modules. Let $A_{1}$ and $A_{2}$ be their principal components. Then the map

$$
\operatorname{Hom}_{\mathcal{O}_{\mathcal{D}}}\left(\underline{M}_{1}, \underline{M}_{2}\right) \rightarrow \operatorname{Hom}_{\mathbb{Z}[\pi \times \pi]}\left(A_{1}, A_{2}\right)
$$

associating with any morphism its effect on the principal components, is an isomorphism.

Proof. Let $f: \underline{M}_{1} \rightarrow \underline{M}_{2}$ be an $\mathcal{O}_{\mathcal{D}}$-morphism. The map (26) associates with $f$ the $\mathbb{Z}[\pi \times \pi]$-homomorphism $f_{1}: \underline{M}_{1}(G / 1)=A_{1} \rightarrow \underline{M}_{2}(G / 1)=A_{2}$. We have the following commutative diagram

$$
\begin{array}{ccc}
\underline{M}_{1}(G / H) & \stackrel{\phi_{H}^{1}}{\rightarrow} & A_{1}^{H} \\
\downarrow f_{H} & & \downarrow f_{1}^{H} \\
\underline{M}_{2}(G / H) & \stackrel{\phi_{H}^{2}}{\rightarrow} & A_{2}^{H}
\end{array}
$$

in which $\phi_{H}^{1}$ and $\phi_{H}^{2}$ are isomorphisms. Thus, we see that the homomorphism $f_{H}$ is uniquely determined by the restriction $f_{1}^{H}$ of $f_{1}$ onto $A_{1}^{H}$.

Corollary 6.2.9. Let $\underline{C}_{*}$ be a chain complex of principal $\mathcal{O}_{\mathcal{D}}$-modules and let $\underline{M}$ be a principal $\mathcal{O}_{\mathcal{D}}$-module. Then the canonical map

$$
\operatorname{Hom}_{\mathcal{O}_{\mathcal{D}}}\left(\underline{C}_{*}, \underline{M}\right) \rightarrow \operatorname{Hom}_{\mathbb{Z}[\pi \times \pi]}\left(C_{*}, M\right)
$$

is an isomorphism of chain complexes. Here $C_{*}=\underline{C}_{*}(G / 1)$ is the principal component of $\underline{C}_{*}$ and $M=\underline{M}(G / 1)$ is the principal component of $\underline{M}$.

Proof. This follows from Lemma 6.2.8.

Corollary 6.2.10. Suppose that the group $\pi$ is principal. Let $C_{*}$ be the chain complex of left $\mathbb{Z}[\pi \times \pi]$-modules consisting of principal components of a projective $\mathcal{O}_{\mathcal{D}}$-resolution of $\underline{\mathbb{Z}}$. Then the natural map

$$
H_{\mathcal{D}}^{n}\left(\pi \times \pi ; \underline{I}^{n}\right) \rightarrow H^{n}\left(\operatorname{Hom}_{\mathbb{Z}[\pi \times \pi]}\left(C_{*}, I^{n}\right)\right),
$$


is an isomorphism.

Proof. We apply Corollary 6.2.9 to a $\mathcal{O}_{\mathcal{D}}$-free resolution of $\underline{\mathbb{Z}}$ noting that under our assumptions the Bredon module $\underline{I}^{n}$ is principal (by Corollary 6.2.7).

6.3. Note that the complex $C_{*}$ which appears in Corollary 6.2 .10 is a resolution of $\mathbb{Z}$ over the ring $\mathbb{Z}[\pi \times \pi]$ but it is neither free nor projective. Any projective resolution $P_{*}$ admits a chain map $P_{*} \rightarrow C_{*}$ and for any left $\mathbb{Z}[\pi \times \pi]$-module $A$ we have a chain map $\operatorname{Hom}_{\mathbb{Z}[\pi \times \pi]}\left(C_{*}, A\right) \rightarrow \operatorname{Hom}_{\mathbb{Z}[\pi \times \pi]}\left(P_{*}, A\right)$ (which is unique up to homotopy) inducing a welldefined homomorphism

$$
H^{*}\left(\operatorname{Hom}_{\mathbb{Z}[\pi \times \pi]}\left(C_{*}, A\right)\right) \rightarrow H^{*}\left(\operatorname{Hom}_{\mathbb{Z}[\pi \times \pi]}\left(P_{*}, A\right)\right)=H^{*}(\pi \times \pi ; A) .
$$

The homomorphism $\Phi$ (see (14)) is the composition of (27) and (28), taken with a suitable module $A$.

\section{Essential COHOMOlogy Classes}

The following notion was introduced and studied in [17].

Definition 7.0.1. Let $A$ be a left $\mathbb{Z}[\pi \times \pi]$-module. A cohomology class $\beta \in H^{n}(\pi \times \pi ; A)$ is said to be essential if there exists a homomorphism of $\mathbb{Z}[\pi \times \pi]$-modules $\mu: I^{n} \rightarrow A$ such that

$$
\mu_{*}\left(\mathfrak{v}^{n}\right)=\beta .
$$

Here $\mathfrak{v}^{n} \in H^{n}\left(\pi \times \pi ; I^{n}\right)$ denotes the $n$-th power of the canonical class $\mathfrak{v}$.

In [17] the authors constructed a spectral sequence giving a full set of obstructions for a cohomology class to be essential. The first such obstruction is the requirement for the class $\beta \in H^{n}(\pi \times \pi ; A)$ to be a zero-divisor, i.e.

$$
\left.\beta\right|_{\pi}=0 \in H^{n}\left(\pi ;\left.A\right|_{\pi}\right)
$$

where $\pi \subset \pi \times \pi$ denotes the diagonal subgroup; see [17], $\S 5$. The condition (29) is obvious since the canonical class $\mathfrak{v}$ and all its powers $\mathfrak{v}^{n}$ are zero-divisors.

Here we characterise the essential cohomology classes as principal components of Bredon cohomology classes.

Theorem 7.1. Let $A$ be a left $\mathbb{Z}[\pi \times \pi]$-module which is the principal component of an $\mathcal{O}_{\mathcal{D}}$-module $\underline{M}$. Consider the homomorphism

$$
\Phi: H_{\mathcal{D}}^{n}(\pi \times \pi ; \underline{M}) \rightarrow H^{n}(\pi \times \pi ; A)
$$

which associates to a Bredon cohomology class its principal component, see (14).

(1) Any class $\beta \in H^{n}(\pi \times \pi ; A)$ in the image of $\Phi$ is essential.

(2) If the group $\pi$ is principal then the set of essential cohomology classes coincides with the image on $\Phi$. 
Proof. Suppose that $\beta=\Phi(\alpha)$ where $\underline{M} \in H^{n}(\pi \times \pi ; \underline{M})$. By the Universality Theorem 5.1 , there exists a $\mathcal{O}_{\mathcal{D}}$-homomorphism $\mu: \underline{I}^{n} \rightarrow \underline{M}$ such that $\alpha=\mu_{*}\left(\mathfrak{u}^{n}\right)$. On the principal components we obtain a $\mathbb{Z}[\pi \times \pi]$-homomorphism $\mu: I^{n} \rightarrow A$ such that $\mu_{*}\left(\mathfrak{v}^{n}\right)=\beta$. Thus $\beta$ is essential. Here we used Theorem 5.2 stating that the principal component of $\mathfrak{u}^{n}$ is $\mathfrak{v}^{n}$. This proves statement (1).

Suppose now that a cohomology class $\beta \in H^{n}(\pi \times \pi ; A)$ is essential, i.e. $\beta=\mu_{*}\left(\mathfrak{v}^{n}\right)$ where $\mu: I^{n} \rightarrow A$ is a $\mathbb{Z}[\pi \times \pi]$-homomorphism. Let $\underline{M}$ denote the $\mathcal{O}_{\mathcal{D}}$-module

$$
\underline{M}(G / H)=A^{H}=[G / H, A]_{G} .
$$

whose principal component is $A$. Here we view $A$ as a left $G$-set and the brackets $[,]_{G}$ denote the set of $G$-maps. Since we assume that $\pi$ is principal we know that $\mathcal{O}_{\mathcal{D}}$-module $\underline{I}^{n}$ is principal (see Corollary 6.2.7). Applying Lemma 6.2 .8 we obtain a $\mathcal{O}_{\mathcal{D}}$-morphism $\hat{\mu}: \underline{I}^{n} \rightarrow \underline{M}$ having $\mu$ as its principal component. This produces a Bredon cohomology class

$$
\alpha=\hat{\mu}_{*}\left(\mathfrak{u}^{n}\right) \in H_{\mathcal{D}}^{n}(\pi \times \pi ; \underline{M}),
$$

and using Theorem 5.2 we have $\Phi(\alpha)=\mu_{*}\left(\mathfrak{v}^{n}\right)=\beta$.

This completes the proof.

\section{EXAMPLES OF PRINCIPAL GROUPS}

In this section we show that all torsion free hyperbolic groups as well as all torsion free nilpotent groups are principal. Also, we give an example of a non-principal group.

Definition 8.0.1. We say that a group $\pi$ satisfies Property $N$ if, for any $a \in \pi$ and any finite set $S \subset \pi$, the inclusion $a^{n} \in Z(S)$, where $n \geq 1$, implies that $a \in Z(S)$.

Proposition 8.0.2. Any group $\pi$ satisfying Property $N$ is principal.

Proof. We shall use the property (c) from Definition 6.2.4. To show that the group $\pi$ is principal we need to show that for any two finite subsets $S, S^{\prime} \subset \pi$ the group $Z(S) / Z\left(S \cup S^{\prime}\right)$ is either trivial or infinite. This will follow once we show that this group is torsion free. An element of order $n$ in $Z(S) / Z\left(S \cup S^{\prime}\right)$ is represented by an element $a \in Z(S)$ such that $a^{n} \in Z\left(S \cup S^{\prime}\right)$. But then Property $\mathrm{N}$ implies $a \in Z\left(S \cup S^{\prime}\right)$ i.e. $a$ represents the trivial class in $Z(S) / Z\left(S \cup S^{\prime}\right)$.

Proposition 8.0.3. If $\pi$ is a finitely generated torsion free nilpotent group, then $\pi$ satisfies Property $N$ and therefore $\pi$ is principal.

Proof. If $\pi$ is abelian, then $Z(S)=\pi$, so Property $N$ holds tautologically. Suppose inductively that any finitely generated torsion free nilpotent group of class $<r$ satisfies Property $N$. Take $\pi$ of class $r$ and let $a^{n} \in Z(S)$ for some $S$. Denote the quotient of $\pi$ by its centre by $\bar{\pi}=\pi / Z(\pi)$ and note that: (1) The class of $\bar{\pi}$ is $<r$, so $\bar{\pi}$ satisfies Property $N$ and (2) $Z(S)$ maps into $Z(\bar{S})$ under the quotient map $\pi \rightarrow \bar{\pi}$. Then we see that $\bar{a}^{n} \in Z(\bar{S})$ and, by Property $N$, we have $\bar{a} \in Z(\bar{S})$. Let $g \in S$ so that $\bar{g} \in \bar{S}$. Then we see that 
$[\bar{a}, \bar{g}]=1$ and this implies that $[a, g] \in Z(\pi)$. Let's now employ a basic relation among higher commutators (which holds for any group [cf. Hall, 10.2.12]),

$$
[x y, z]=[x,[y, z]][y, z][x, z] .
$$

Recall that we have $\left[a^{n}, g\right]=1$. Expanding $\left[a^{n}, g\right]$ using the relation above gives

$$
\left[a^{n}, g\right]=\left[a^{n-1},[a, g]\right][a, g]\left[a^{n-1}, g\right]=[a, g]\left[a^{n-1}, g\right]
$$

where the last equality follows because $[a, g] \in Z(\pi)$. Repeating this step eventually leads to $1=\left[a^{n}, g\right]=[a, g]^{n}$. Since $\pi$ is torsion free, we have $[a, g]=1$ and $a \in Z(S)$. This completes the inductive step.

Lemma 8.0.4. Let $\pi$ be a torsion free group such that the centraliser $Z(g)$ of any element $g \in \pi-\{1\}$ is cyclic. Then any two centralisers $Z\left(g_{1}\right), Z\left(g_{2}\right)$, where $g_{1}, g_{2} \in \pi-\{1\}$, either coincide $Z\left(g_{1}\right)=Z\left(g_{2}\right)$ or their intersection is trivial, $Z\left(g_{1}\right) \cap Z\left(g_{2}\right)=\{1\}$.

Proof. Let $a_{i} \in Z\left(g_{i}\right)$ be a generator, $i=1,2$. Assume that the intersection $Z\left(g_{1}\right) \cap Z\left(g_{2}\right)$ is not trivial. Then this intersection is an infinite cyclic group. Let $x \in Z\left(g_{1}\right) \cap Z\left(g_{2}\right)$ denote a generator of the intersection. Then

$$
x=a_{1}^{n_{1}}=a_{2}^{n_{2}}
$$

for some $n_{1}, n_{2} \neq 0$. Consider the centraliser $Z(x) \subset \pi$. It is an infinite cyclic group (by our assumption) which contains $a_{1}$ and $a_{2}$ (because of (31)) implying that the elements $a_{1}$ and $a_{2}$ commute. Hence $Z\left(g_{1}\right)=Z\left(g_{2}\right)$.

Lemma 8.0.5. Assume that a group $\pi$ is torsion free and the centraliser of any nontrivial element $g \in \pi$ is cyclic. Then $\pi$ satisfies property $N$ and hence it is principal.

Proof. Let $S \subset \pi$ be a finite subset. By Lemma 8.0.4, if $Z(S)$ is nontrivial then $Z(S)=$ $Z(g)$ for some $g \in \pi-\{1\}$. If $a^{n} \in Z(S)$ then $a^{n} \in Z(a) \cap Z(g)$. We know that the centralisers $Z(a)$ and $Z(g)$ either coincide or have trivial intersection. If $Z(a)=Z(g)$ then $a \in Z(g)=Z(S)$. In the case $Z(a) \cap Z(g)=1$ we obtain $a^{n}=1$ and hence $a=1$ since $\pi$ is torsion free.

Corollary 8.0.6. Any torsion free hyperbolic group is principal.

Proof. This follows from Lemma 8.0.5 since in a torsion free hyperbolic group the centraliser of any non-unit element is cyclic.

As example of a group that is not principal we have the following:

Example 8.0.7. Consider the fundamental group $K$ of the Klein bottle,

$$
K=\left\langle c, d ; c^{2}=d^{2}\right\rangle
$$

Denote $z=c^{2}=d^{2}$; this element generates the centre $Z \subset K$. Denote $x=c d, y=d c$. Any element of $K$ can be uniquely written in one of the four forms

$$
x^{k} z^{l}, y^{k} z^{l}, x^{k} z^{l} c, y^{k} z^{l} d, \quad k, l \in \mathbb{Z} .
$$


Relations:

$$
\begin{gathered}
x y=y x=z^{2} \\
c x=y c \\
d x=y d \\
c y=x c \\
d y=x d
\end{gathered}
$$

We see that the centraliser of $x$ is the subgroup generated by $x, y$ and $z$. Note that $Z(x) \subset K$ is normal. Besides, $c \notin Z(x)$ while $c^{2}=z \in Z(x)$. This shows that $K$ does not have property $N$.

Besides, the centraliser of $x y=z^{2}$ is the whole group $K$. In this case the group $K / K \cap$ $Z(x)=K / Z(x)$ is $\mathbb{Z}_{2}$. Consider the following two subgroups $H, H^{\prime} \subset K \times K$. Let $H=\Delta \subset K \times K$ be the diagonal and let $H^{\prime}$ be $H^{\prime}=\left\{\left(a, x a x^{-1}\right) ; a \in K\right\}$. Then $H \cap H^{\prime}=\{(a, a) ; a \in Z(x)\}$ and hence $H / H \cap H^{\prime} \simeq K / Z(x) \simeq \mathbb{Z}_{2}$. We conclude that the fundamental group of the Klein bottle $K$ is not principal.

\section{REFERENCES}

[1] A. Boudjaj and Y. Rami, On spaces of topological complexity two, arXiv 1607. 05346v2, 25 July 2016.

[2] G. Bredon, Equivariant cohomology theories Lecture Notes in Mathematics, No. 34 Springer-Verlag, Berlin-New York 1967.

[3] K. S. Brown, Cohomology of groups, Graduate Texts in Mathematics, vol. 87, Springer-Verlag, New York, 1982.

[4] D. Cohen and G. Pruidze, Motion planning in tori, Bull. Lond. Math. Soc. 40 (2008), 249-262.

[5] D. Cohen and L. Vandembroucq, Topological complexity of the Klein bottle, J Appl. and Comput. Topology (2017). https://doi.org/10.1007/s41468-017-0002-0, arXiv:1612.03133.

[6] A. Costa and M. Farber, Motion planning in spaces with small fundamental groups, Commun. Contemp. Math. 12 (2010), no. 1, 107-119.

[7] O. Cornea, G. Lupton, J. Oprea, and D. Tanré, Lusternik-Schnirelmann Category, Mathematical Surveys and Monographs, vol. 103, American Mathematical Society, Providence, RI, 2003.

[8] A. Dranishnikov and Y. Rudyak, On the Berstein-Svarc theorem in dimension 2, Math. Proc. Cambridge Philos. Soc. 146 (2009), no. 2, 407-413.

[9] A. Dranishnikov, The topological complexity and the homotopy cofiber of the diagonal map for nonorientable surfaces, Proc. Amer. Math. Soc. 144 (2016), no. 11, 4999-5014.

[10] S. Eilenberg and T. Ganea, On the Lusternik-Schnirelmann category of abstract groups, Ann. of Math. (2) 65 (1957), 517-518.

[11] M. Farber, Topological complexity of motion planning, Discrete Comput. Geom. 29 (2003), no. 2, 211-221.

[12] M. Farber, Instabilities of robot motion, Topology Appl. 140 (2004), no. 2-3, 245-266.

[13] M. Farber, S. Tabachnikov and S. Yuzvinsky, Motion planning in projective spaces, International Mathematics Research Notices 2003, No. 34, pp. 1853 - 1870.

[14] M. Farber, Topology of robot motion planning, in: Morse theoretic methods in nonlinear analysis and in symplectic topology, NATO Sci. Ser. II Math. Phys. Chem., vol. 217, Springer, Dordrecht, 2006, pp. $185-230$.

[15] M. Farber, Invitation to topological robotics, Zurich Lectures in Advanced Mathematics, European Mathematical Society (EMS), Zürich, 2008. 
[16] M. Farber, Configuration Spaces and Robot Motion Planning Algorithms, in: Combinatorial and Toric Topology, A. Darby, J. Grbic, Z. Lü and J. Wu editors, Lecture Notes Series, IMS, National University of Singapure, 2017, pp. 263 - 303.

[17] M. Farber, S. Mescher, On the topological complexity of aspherical spaces, preprint arXiv:1708.06732.

[18] M. Farber, S. Yuzvinsky, Topological robotics: subspace arrangements and collision free motion planning, Geometry, topology, and mathematical physics, 145-156, Amer. Math. Soc. Transl. Ser. 2, 212, Adv. Math. Sci., 55, Amer. Math. Soc., Providence, RI, 2004.

[19] M. Fluch, PhD Thesis: On Bredon (Co-)Homological Dimensions of Groups, University of Southhampton (2011).

[20] M. Grant, Topological complexity, fibrations and symmetry, Topology Appl. 159 (2012), no. 1, 88-97.

[21] M. Grant, G. Lupton and J. Oprea, New lower bounds for the topological complexity of aspherical spaces, Topology Appl. 189 (2015), 78-91.

[22] M. Grant, G. Lupton and J. Oprea, Spaces of topological complexity one, Homology, Homotopy and Applications, vol. 15(2) (2013) 73-81.

[23] M. Grant, G. Lupton, and J. Oprea, A mapping theorem for topological complexity, Algebr. Geom. Topol. 15(2015), no. 3, 1643-1666.

[24] M. Grant and D. Recio-Mitter, Topological complexity of subgroups of Artin's braid groups, arXiv:1607.04830.

[25] A. Hatcher, Algebraic topology, Cambridge University Press, Cambridge, 2002.

[26] D. Husemoller, Fibre Bundles, McGraw-Hill Company, 1966.

[27] W. Lück, Survey on classifying spaces for families of subgroups, Infinite groups: geometric, combinatorial and dynamical aspects, 269-322, Progr. Math., 248, Birkhäuser, Basel, 2005.

[28] W. Lück, Transformation groups and algebraic K-theory, Lecture Notes in Math, v. 1408, Springer Verlag, Berlin, 1989.

[29] S. Mac Lane, Homology, Springer-Verlag, 1995.

[30] J.P. May, Equivariant homotopy and cohomology theory, AMS Regional Conference Series in Mathematics 91,1996.

[31] G. Mislin, Equivariant K-Homology of the Classifying Space for Proper Actions, in G. Mislin and A. Valette, Proper group actions and the Baum-Connes Conjecture, Birkhauser, 2003.

[32] J. Milnor, On spaces having the homotopy type of a CW complex, Trans. Amer. Math. Soc. 90 (1959), $272-280$.

[33] Y. Rudyak, On topological complexity of Eilenberg-MacLane spaces. Topology Proceedings 48 (2016), $65-67$.

[34] A. Schwarz, The genus of a fiber space, Amer. Math. Soc. Transl. Ser. 2 55(1966), 49-140.

[35] T. tom Dieck, Transformation groups, De Gruyter Studies in Math. 8, 1987.

[36] G. Whitehead, Elements of Homotopy Theory, Grad. Texts in Math. 61 Springer-Verlag (1978).

School of Mathematical Sciences, Queen Mary, University of London, London, E1 4NS, United Kingdom

Email address: m.farber@qmul.ac.uk

Institute of Pure And Applied Mathematics, University of Aberdeen, Aberdeen AB24 3UE, United Kingdom

Email address: mark.grant@abdn.ac.uk

Department of Mathematics, Cleveland State University, Cleveland OH 44115, U.S.A.

Email address: g.lupton@csuohio.edu

Department of Mathematics, Cleveland State University, Cleveland OH 44115, U.S.A.

Email address: j.oprea@csuohio.edu 